\title{
POLARIZATIONAL EFFECTS AND THE CRITICAL BEHAVIOUR OF SYSTEMS WITH COULOMBIC INTERACTIONS
}

\author{
V. L. Kulinskii, N. P. Malomuzh \\ Odessa National University, Department of Theoretical Physics, \\ 2 Dvoryans'ka Str., Odessa, UA-270100, Ukraine \\ (Received April 17, 2002; reseived in final form July 2, 2002)
}

\begin{abstract}
The review is devoted to the critical behaviour of the systems with Coulombic interactions. The importance of polarizational effects near the critical point in such systems is emphasized. It is shown that polarizational interactions essentially renormalize the initial effective Landau-Ginzburg Hamiltonian of the systems. The dipole fluid model as the basic one for the criticality of ionic liquids (molten salts like $\mathrm{NaCl}$ ) is considered. It is shown that taking into account the internal degrees of freedom, namely the rotational ones of an ionic pair allows to formulate the new approach to the problem about the physical size of a pair. The estimates for the critical parameters within dipole fluid model are given. Possible scenarios for the criticality of the system with Coulombic interaction are proposed. The possibility of formation of inhomogeneous states in the critical region is discussed. Some specific effects caused by the polarizational induced interaction between order parameter and the charge density fluctuation characteristic for the system with the direct Coulombic interactions are discussed. Some new problems connected with the Coulombic criticality are outlined.

Key words: critical point, coulombic interaction, polarizational effects.
\end{abstract}

PACS number(s): 05.70.Jk, 64.60.Fr, 64.70.Fx

\section{Contents}

Critical behaviour of the systems with Coulombic interactions

A Types of systems with Coulombic interactions and experimental facts . . . 52

B Mean-field theories of RPM ..... 53

C Renormalization group analysis and Kosterlitz-Thouless transition . . .

D Qualitative analysis of the critical behaviour of the dipole liquid . . . . . . . . . 56

E The dissociation of the rotating dipole liquid .............. 58

Polarizational interactions

A General theory of polarizational interactions . . . . . . . . . .

B Effective Landau-Ginzburg Hamiltonian for ionic liquids

C The effective Landau-Ginzburg Hamiltonian for electrolyte solutions . . . . .

D Canonical form for the effective Hamiltonian

Influence of the polarizational effects on the critical behaviour

A Ginzburg number for the ionic liquids .

B Ginzburg criterion for electrolyte solutions .................

\section{Specific effects}

A Fluctuation-induced shift of the critical point in solution of electrolytes . . . . .

B Asymmetry of a binodal for electrolyte solutions
C Conductivity of the electrolytes

V

Conclusion

74

\section{INTRODUCTION}

In recent years critical phenomena in systems with Coulombic interaction between particles have been the object of many review papers [1-12]. From them it follows that the description of the thermodynamics of ionic system is far from complete understanding. In contrast to the critical behaviour in molecular liquids both mean field and scaling analysis for ionic liquids (IL) have yet to be resolved. The characteristic problem for IL is the construction of suitable mean-field theory which would give the reliable phase diagram and estimates for non universal quantities like Ginzburg number, critical amplitudes, etc. With the help of such self-consistent mean field approach the influence of interplay between direct Coulomb and specific interactions on the type of the critical behaviour could be investigated. The problem of manifestation of peculiarities of interparticle interactions in non-universal characteristics of phase transitions becomes very important.

The experimental study of the critical phenomena in systems with Coulombic interactions revealed unexpected results. First of all, such systems demonstrate essential differences in their critical behaviour. The main question here is about the existence of the crossover region, which for the systems of early studies [1] was found to be of the order $\tau=\frac{T-T_{c}}{T_{c}} \approx 10^{-4}$ [13]. A recent review [11] of the experimental results of the lasts two decades states that "...there are several sets of highly accurate data which, however, contradict fundamentally". 
There are suspicions that some systems show tricritical behaviour $[16,15]$. In fact, it means that 1) Ginzburg number for many systems with Coulombic interaction (CI) is abnormally small or 2) for some systems the Coulombic interactions lead to the quantitative change of the critical behaviour at all $\tau$. On the other hand the nature of low values for the dimensionless critical densities and the temperature for molten salts like $\mathrm{NaCl}$ needs clarification. In particular, the question about the degree of ionization of the system near the critical point remains open. The clear answer to these questions is one of the main problem for the modern theory of critical phenomena.

Here we should note that in fact there is another type of the systems with Coulombic interaction - liquid metals (alkali metals and mercury). It is well grounded experimentally that liquid metals belong to the Ising model universality class of the critical behaviour [14]. Moreover their critical behaviour is characterized by the same Ginzburg number value as that in molecular liquids like Ar. Ne, etc. The difference in interparticle interaction causes the difference in nonuniversal critical amplitudes. The asymmetry of a coexistence curve here serves as the characteristic example. In monovalent alkali metals with lowest $T_{c}(\mathrm{Cs}, \mathrm{Rb})$ a strong asymmetry takes place. In contrast to it the liquid-vapor equilibrium curve for divalent $\mathrm{Hg}$ looks nearly symmetrical. This difference is connected with existence of metal-insulator transition (MIT), which leads to a significant difference in interparticle potential for vapour and liquid phases in alkali metals. Alkali metals in liquid phase near critical point are indeed metals with nearly free electron transport properties, while for $\mathrm{Hg}$ MIT happens at higher density $\rho_{\mathrm{MI}}>\rho_{c}$. Thus, with respect to the conductivity values, liquid mercury near critical region is treated as slightly ionized fluid rather than liquid metal [14].

From the thermodynamic point of view the position of the vapour-liquid critical point is determined by the conditions:

$$
\left(\frac{\partial p}{\partial v}\right)_{T}=0 \quad\left(\frac{\partial^{2} p}{\partial v^{2}}\right)_{T}=0
$$

where $p, v, T$ are the pressure, the specific volume and the temperature correspondingly. These conditions do not depend on the specific character of interparticle interactions. Within the Landau approach for the free energy:

$$
F=F_{0}+V\left[A_{2} \phi^{2}+A_{3} \phi^{3}+A_{4} \phi^{4}+\ldots\right]
$$

from (1) it follows that only $A_{2}$ and $A_{3}$ can vanish at the critical point.

The fluctuations of the order parameter $\phi$ and the charge density change the initial values of coefficients $A_{i}$. For the non-ionic liquids these variations are not drastic in the sense that $A_{4}$ remains positive. However, for the IL a new possibility may be realized. Due to the associationdissociation process near the critical point the appearance of spatially inhomogeneous state is possible. More exactly, the drops of fully dissociated liquid can appear in dipole fluid and vise versa. Such a possibility will change essentially the type of the critical behaviour.

For the systems with CI there is one more important cause which influences the character of the phase transition. Due to strong fluctuations of the order parameter the fluctuations of the dielectric permittivity also abnormally grow. As a result the interaction energy $U_{c h}$ of the charge fluctuations $\delta \rho$ is not described by the simple formula:

$$
U_{\mathrm{ch}}=\frac{1}{2 \epsilon_{0}} \iint d \mathbf{r} d \mathbf{r}^{\prime} \frac{\delta \rho(\mathbf{r}) \delta \rho\left(\mathbf{r}^{\prime}\right)}{\left|\mathbf{r}-\mathbf{r}^{\prime}\right|}
$$

characteristic for the systems with quasiconstant values $\epsilon_{0}$ of the dielectric permittivity. In the considered case the interaction energy will be more complex functional of type:

$$
U_{\mathrm{ch}}=\frac{1}{2} \iint d \mathbf{r} d \mathbf{r}^{\prime} \delta \rho(\mathbf{r}) \delta \rho\left(\mathbf{r}^{\prime}\right) \sum_{i=0}^{\infty} \frac{1}{n !} \int K_{n}\left(\mathbf{r}, \mathbf{r}^{\prime} \mid \mathbf{q}_{1}, \ldots, \mathbf{q}_{n}\right) \delta \epsilon\left(\mathbf{r}_{1}\right) \ldots \delta \epsilon\left(\mathbf{q}_{n}\right) d \mathbf{q}_{1} \ldots d \mathbf{q}_{n}
$$

where $K_{0}=\frac{1}{\epsilon_{0} \mid \mathbf{r}-\mathbf{r}^{\prime}}$. The explicit form for the kernels $K_{n}$ should be determined from the Poisson equation for a medium with inhomogeneous dielectric permittivity. The interactions, generated by the functions $K_{n}, n \geq 1$, we will name as polarizational ones. As a consequence of direct connection between $\delta \epsilon$ and $\phi$ the polarizational terms to $U_{\text {ch }}$ renormalize also the corresponding coefficients of the Landau-Ginzburg Hamiltonian (LGH):

$$
H_{\mathrm{LG}}[\phi]=\int d V\left[\frac{b}{2}(\nabla \phi(\mathbf{r}))^{2}+\frac{1}{2} a_{2} \phi^{2}(\mathbf{r})+\frac{1}{4} a_{4} \phi^{4}(\mathbf{r})+\ldots\right]
$$


It is very essential to note that the renormalization may be effectively amplified by the relatively small value of $a_{4}$, which, as it appears, takes place in IL in comparison with molecular ones [6]. The role of the polarizational effects in the critical phenomena and construction of the Landau-Ginzburg Hamiltonian for the solutions of electrolytes and ionic liquids were investigated in [17] and [18].

In this review we present the main results of investigations of critical phenomena in systems with Coulombic interactions with special accent on the role of the polarizational effects. Our attention will be focused on the following main questions:

1. the nature of the critical point in molten salts like $\mathrm{NaCl}$;

2. the definition and discussion of the main properties of polarizational interactions in electrolyte solutions and IL;

3. the concentration dependence of the Ginzburg number for electrolytes near their vapour-liquid critical points;

4. the estimate of the Ginzburg number for IL like $\mathrm{NaCl}$;

5. the nontrivial fluctuational shift of the critical point locus for the electrolytes;

6. the new phase which appearance is possible near the critical point;

7. dielectric properties of ionic fluids.

The material is arranged in the following way:

1. Chapter I is devoted to the general facts on the critical behaviour of different systems with CI. Here we present also the alternative basic models for the description of the Coulombic criticality in molen salts: the RPM and dipole fluid (DF).

2. The definition and general discussion of the polarizational interactions are in Chapter II. Our consideration will be restricted by the discussion of the polarizational effects mostly on the mean field level. The explicit form of the LGH for the systems of interest is proposed.

3. The dependence of the Ginzburg number on the relevant parameters of the system with CI is discussed in Chapter III.

4. In Chapter IV such important characteristics as conductivity and the asymmetry of the binodal are analyzed.

\section{CRITICAL BEHAVIOUR OF THE SYSTEMS WITH COULOMBIC INTERACTIONS}

\section{A. Types of systems with Coulombic interactions and experimental facts}

Critical phenomena in fluids with nondispersive Coulombic interactions have attracted much attention in the last ten years owing to a diversity of experimental results. Early experimental results of $[2,13,19]$ ) favored mean-field type of the critical behaviour while recent studies $[20,21]$ confirmed Ising-like asymptotics for the systems under investigation.

The variety of systems studied experimentally can be divided into two groups. Below we will name them as Type I and Type II systems.

The systems of Type I are those where as is suggested the Coulombic criticality takes place. The latter is characterized by a pronounced narrowing of the fluctuation region with probable mean field or the tricritical point variants for critical behaviour. At least the apparent crossover from classical to Ising-like critical behaviour is expected. This group includes pure ionic fluids with liquid-gas phase separation such as molten alkali halides $\mathrm{NaCl}, \mathrm{KCl}$, etc. [3,5]. The liquid-vapor critical point of these systems is located at very high temperatures $\left(T_{c} \simeq 10^{3} \mathrm{~K}\right)$. This makes the experiments very difficult. In addition for such systems the number of thermodynamic degrees of freedom allows tricriticality as rather degenerated but not generic case.

It is expedient to introduce common dimensionless density and temperature [9]:

$$
\rho^{*}=n a^{3}, \quad T^{*}=\frac{\epsilon k_{B} T}{q^{2} / a}
$$

where $a$ is the ionic diameter, $n=\frac{N}{V}$ is the total number density, $q$ is the absolute value of the charge.

Typical values of $\rho$ near the critical point is less than 0.1 , and usually it is connected with small association taken into account with the help of thermodynamic perturbation theory. However, the reliability of such type estimates is not quite clear. Therefore the development of the alternative approach grounded on the dipole fluid model seems to be expedient. In the present paper we estimate the critical characteristics of the ionic molten in the framework of the dipole fluid approach. We will also show that the change of molecular parameters due to rotations influences essentially the location of the critical point.

From this point of view the ionic (quasi)binary mixtures with "room" critical temperatures $\left(T_{c} \simeq 300 \mathrm{~K}\right)$ of decomposition used in the experiments by Pitzer and coworkers are more suitable $[2,19,22,23]$ and widely used. Tricritical variant of the critical behaviour for multicomponent systems cannot by excluded a priory. The experimental results are interpreted in $[16,15]$ within this point of view. The possibility of tricritical nature of observed peculiarities was also discussed in [6,7]. For such 
a conjecture to be valid the additional scale competitive with correlation length of an order parameter must exist [6]. For example, in polymer blends the apparent second scale is the size of a molecule [24], which may lead to the crossover. The physical meaning of its analogue, if there exists one, for ionic melts and electrolyte solution is not clear [16].

Analogous problems arise for the second group, which includes dilute solutions of electrolytes, such as aqueous electrolytes $\left(\mathrm{H}_{2} \mathrm{O}+\mathrm{NaCl}\right.$ etc. $)[25,26]$, and quasibinary solutions [16]. Here the admixture of ions changes the parameters of the critical point of pure solvent. Thus the critical behaviour of a pure solvent is perturbed by the Coulombic subsystem of ions. The critical behaviour of these systems may exhibit a crossover from mean-field to Ising-like asymptotics within the temperature interval of an experiment. In particular, experimental data for some solutions were fitted with mean-field exponential law up to $\tau \approx 10^{-4}[13]$, i.e., the fluctuation region is much less in comparison with that for simple liquids $(G i \approx 0.1 \div 1)$. This is in apparent contrast to the results of $[27]$ where $G i$ decreases insignificantly if the ionicity parameter grows.

$$
I=\frac{q^{2} / a}{k_{\mathrm{B}} T_{c}^{(0)}}
$$

Here $T_{c}^{(0)}$ is the critical temperature of the solvent, provided that the critical behaviour belongs to Ising-model universality class. The ionicity parameter ( 7 ) obviously shows the relative strength of Coulombic and solvophobic, short range forces. The last forms Ising like critical behaviour of neutral system. Though the account of direct Coulombic interaction in [27] was performed pertubatively without accounting the pair formation. It is clear that in such a way there is no substantial interaction between Coulombic and molecular subsystems. Thus due to simple screening of the Coulombic interaction no drastic changes would be expected for $G i$ in such an approach.

To process such data, different assumptions in $[28,29]$ and [30] were considered. These affected the conclusions about the existence of the crossover in such systems. The results of [30] show the nonclassical behaviour through the temperature interval without any crossover. In $[28,29]$ the experimental data were fitted by a classical EOS.

The basic model for these systems is the restricted primitive model (RPM). The RPM is the system of equal number of positive and negative charges $\pm q$ of the diameter $a$, immersed in a homogeneous medium of dielectric permittivity $\epsilon$. It can be expected that such model is adequate for ionic molten salts. Various theoretical approximations and numerical simulations predict the liquidvapour critical point at very low dimensionless temperatures and densities $[6,31,12]$. The nature of critical fluctuations in this model are much less studied. The main difficulty here is the interaction between number density and charge fluctuations.

\section{B. Mean-field theories of RPM}

The consideration of the critical behaviour in such is based on the so called Primitive Model, which is defined by the classical potential:

$$
U(r)= \begin{cases}\frac{q_{i} q_{i}}{r_{i j}}, & r_{i j} \geq \sigma=\frac{\sigma_{+}+\sigma_{-}}{2} \\ \infty, & r_{i j}<\sigma\end{cases}
$$

Note that due to classical character of the model, the masses of the charges plays no role. If the symmetry between charges is 'broken', one gets the extended, 'unrestricted' version of the model (see [11] and references therein). The restricted version of this model deals with specific case of equisized ions with the same absolute value of charge. The mean field theory for the critical behaviour of such a system is mainly constructed in three ways: 1) thermodynamic perturbation theory [6]; 2) pair correlation function analysis [7]; 3) computer simulation of EOS $[32,33]$. The location of the critical point for RPM varies in dependence of the interactions included into free energy. Typical values of critical point locus are:

$$
\rho_{c}^{*} \approx 0.02 \div 0.035 \quad T_{c}^{*} \approx 0.04 \div 0.06
$$

The MC simulations give to some extent contradictory results for the position of the critical point as well as for the type of the critical behaviour. For the lasts the careful analysis of the finite size scaling effects is needed. This probably the main cause for the differences between the results based on the Gibbs ensemble [34] which locate the critical density at almost the same value as in (9). Others based on the extrapolation of the dependence of the cell size $L$ suggest significantly higher densities $\rho_{c}^{*} \approx 0.07 \div 0.08[32]$.

There have been proposed many approaches to investigate the criticality of the RPM. The first approach is the thermodynamic perturbation theory. The main goal of it is to incorporate as much physically relevant effective interactions into thermodynamic potential (free energy) as possible. In particular, it is based on the generalization of classical Debye-Hückel (DH) theory [35] for electrolytes. It is assumed that the free energy of the system per unit volume has the form:

$$
f(\rho, T)=\sum_{i} f^{(\mathrm{id})}\left(\rho_{i}, T\right)+f^{(\mathrm{ex})}\left(T, \rho_{\mathrm{ch}}, \rho_{d}\right)
$$

where $f^{(\text {id) }}\left(\rho_{i}, T\right)$ is the ideal gas term for neutral molecules or ions, $\rho_{i}$ is the number density of the charges if $i=+,-$ and dipole pairs at $i=d$. The second term 


\section{L. KULINSKII, N. P. MALOMUZH}

$f^{(\mathrm{ex})}\left(T, \rho_{\mathrm{ch}}, \rho_{d}\right)$ is the exchange part of the free energy which can be represented in the form:

$$
f^{(\mathrm{ex})}\left(T, \rho_{\mathrm{ch}}, \rho_{d}\right)=f^{(n)}\left(T, \rho_{\mathrm{ch}}, \rho_{d}\right)+f^{(\mathrm{ch})}\left(T, \rho_{\mathrm{ch}}, \rho_{d}\right)
$$

where $f^{(n)}\left(T, \rho_{\mathrm{ch}}, \rho_{d}\right)$ is the part including hard core effects and all dispersive interactions of neutral molecules and ions. In particular $f^{(n)}\left(T, \rho_{\mathrm{ch}}, \rho_{d}\right)$ can be taken in a form leading to Carnahan-Starling EOS [36].

Direct Coulombic interactions are take into account by the second term $f^{(\mathrm{ch})}\left(T, \rho_{\mathrm{ch}}, \rho_{d}\right)$. It explicitly depends on the charges $q_{i}$ via characteristic length scale - screening length. The simplest form of this term is given by the classical Debye-Hückel theory, where for small charge densities:

$$
\beta f^{(\mathrm{ch})}\left(T, \rho_{\mathrm{ch}}, \rho_{d}\right) \approx \beta f^{(D H)}(T, \Gamma)=-\frac{\Gamma^{3}}{12 \pi}+o\left(\Gamma^{3}\right)
$$

where $\beta=\frac{1}{k_{\mathrm{B}} T}$. The parameter $\Gamma$ is the inverse screening length, and for the RPM it equals to:

$$
\Gamma^{2}=4 \pi q^{2} \frac{\rho_{\mathrm{ch}}}{T^{*}}
$$

The classical Debye-Hückel (DH) model was improved $[9,37]$ and extended to higher dimensions of space [38]. The generalizations of the expression (12) for the free energy includes the finite size of the ions:

$$
\beta f^{(\mathrm{DH})}\left(T, \rho_{\mathrm{ch}}, \rho_{d}\right)=-\frac{1}{4 \pi}\left(\ln (1+x)-x+\frac{1}{2} x^{2}\right)
$$

and ion-dipole interaction

$$
f^{(\mathrm{di})}=\frac{\Gamma^{2} a a_{1}}{T^{*} a_{2}} \rho_{d} \omega\left(a_{2} \Gamma\right)
$$

where

$$
x=a \Gamma
$$

and

$$
\omega(x)=\frac{3}{x^{4}}\left[\ln \left(1+x+\frac{1}{3} x^{2}\right)-x+\frac{1}{6} x^{2}\right] .
$$

The values of $\rho_{\text {ch }}$ and $\rho_{d}$ are determined according to :

$$
\rho_{\mathrm{ch}}=\Delta \rho^{*}, \quad \rho_{d}=(1-\Delta) \frac{\rho^{*}}{2},
$$

where $\Delta$ is the degree of ionization. The mass action law relates $\Delta$ with the pair association constant $K(T)$ [39]:

$$
2 \frac{1-\Delta}{\Delta^{2}}=\rho^{*} K(T) \exp \left(\beta \mu_{+}^{(\mathrm{ex})}+\beta \mu_{-}^{(\mathrm{ex})}-\beta \mu_{d}^{(\mathrm{ex})}\right),
$$

where $\mu_{i}^{(\mathrm{ex})}=\mu_{i}-\mu_{i}^{(\mathrm{id})}$ is the excess chemical potential for ions $(i=+,-)$ or dipole pair $(i=d)$. The expressions for the chemical potentials are obtained in standard way [39]. As a result they are the functions of $\Delta$. Therefore equation (17) is one for $\Delta$. Also note that for the RPM $\mu_{+}=\mu_{-}$.

The formation of associated pairs of the ions is taken into account within the Bjerrum's concept [40]. The Bjerrum criterion of the pair is based in the simplest modeling $K(T)$ as the classical partition function of dipole configuration, characterized by the only parameter the distance between ions:

$$
\begin{aligned}
K\left(T^{*}\right) & =\int_{a<r<R} \exp \left(\beta \frac{q^{2}}{r}\right) d \mathbf{r} \\
& =4 \pi a^{3} \int_{1}^{R^{*}} x^{2} \exp \left(\frac{1}{x T^{*}}\right) d x .
\end{aligned}
$$

The upper limit of integration is the Bjerrum length which corresponds to the minimum of the integrand and is given by the Bjerrum energetic condition:

$$
\frac{q^{2}}{R}=2 k_{\mathrm{B}} T
$$

so that:

$$
R^{*}=\left\{\begin{array}{cl}
\frac{1}{2 T^{*}} & \text { if } T^{*} \leq \frac{1}{2} \\
1 & \text { if } T^{*}>\frac{1}{2}
\end{array}, \quad R^{*}=R / a .\right.
$$

A more sophisticated approach to modeling the association constant (18) was elaborated in [41]. It was based on the consistency of mass action law with virial expansions for EOS of ionic system up to $\rho_{\mathrm{ch}}^{5 / 2}$ order. But the difference between association constant (18) and that obtained in [41] for the interval of densities and temperatures of interest $\left(T^{*}<0.1, \rho^{*}<0.1\right)$ appeared to be less than $1 \%$. The coordinates of the critical point obtained in such an approach are

$$
T_{c}^{*}=0.055, \quad \rho_{c}^{*}=0.026, \quad P_{c}^{*}=3.6 \cdot 10^{-4} .
$$

For the reviews of the results see $[6,12]$.

The second approach is based on construction of pair the correlation functions. The closure of OrnsteinZernike relation for pair correlation function and subsequent construction of the thermodynamic potential and EOS are used. The above mentioned Debye-Hückel approximation can be obtained within this framework too. 
This is a classical result of statistical physics (see, e.g., [39]).

In this approach the Mean Spherical Approximation (MSA) [42] for pair correlation functions is widely used. Within the latter the equation for the pair correlation function in some cases [43] can be solved exactly. The ion contribution to the free energy in this approximation is:

$$
f^{(\mathrm{ch})}=-\frac{1}{12 \pi a^{3}}\left(2+6 x+3 x^{2}-2(1+2 x)^{3 / 2}\right) .
$$

The association effects are taken into account as has been outlined above with the help of the chemical association theory $[44,45]$. The coordinates of the critical point for MSA-based models with the inclusion of association [46] typically are:

$$
T_{c}^{*}=0.07, \quad \rho_{c}^{*}=0.022, \quad P_{c}^{*}=2.0 \cdot 10^{-4} .
$$

The results obtained within this approach can be found in $[7]$.

The main difficulty of this approach as well as any other connected with using approximations for lower correlation functions is the necessity to satisfy many thermodynamical identities. For Coulomb systems there are additional identities known as sum rules [47]. Usually only the relations for the first (local electroneutrality conditon) and second moments (Stillinger-Lovett sum rule) are fulfilled (e.g., for DH or MSA). The absence of reliable mechanism within this approach which explicitly takes into account all thermodynamic identities results in violation of some exact inequalities as was shown in [48].

Despite obvious progress in matching the coordinates of the critical point obtained in such mean-field thermodynamic approach with those of MC calculations the modified DH theory still confronts the difficulty in the explanation of small values of $G i$. Indeed, once the proper mean field theory is given, the corresponding LGH can be constructed, thus providing the satisfactory estimates for both critical point locus and the width of fluctuational region.

\section{Renormalization group analysis and Kosterlitz-Thouless transition}

The nature of critical fluctuations in the RPM which is directly connected with Coulombic criticality has not been thoroughly studied. The main difficulty here is the interaction between the number density fluctuations and the ones of charge. For the Coulomb gas of point particles the coefficients $a_{2 n}$ of the effective LGH are:

$$
a_{2 n}=(-1)^{n+1}
$$

with $a_{4}<0$. This model is isomorphic to the so-called sine-Gordon field theory [49].
The RPM differs from gas model since: 1) the ions have finite sizes 2) there is nonzero association in the system. Therefore, to derive the proper LGH these features should be included.

The renormalization group (RG) analysis of critical fluctuations in Coulomb gas was done in [50]. The hard core effects were included and treated with the help of Hubbard-Scofield method [51] (see also [36]). Note that the inclusion of hard core effects leads to the density dependence of the coefficients of the effective LGH. To consider the possibility of vanishing $a_{4}$ the effective LGH in the " $\phi$ " approximation was used, where $\phi$ is the field variable conjugated to the charge density. It was shown that the investigated model may exhibit either a firstorder transition or Ising-like critical behaviour depending on the starting values of the LGH coefficients. Two types of the critical behaviour mentioned above are formed due to the existence of a tricritical surface in the space of the coefficients of the Hamiltonian. The behaviour depends on the starting value of $a_{6}>0$. The LGH moves either to the sector where $a_{4}<0$ or to Ising fixed point with $a_{4}>0$ in the space of the LGH coefficients. The estimate for the width of the fluctuational region was not given. In addition the value of the coefficient $a_{6}$ was considered as an arbitrary parameter of the theory $\left(a_{6}>0\right)$. Though for lower densities $(0.01<\rho<0.07)$ all the coefficients at $\phi^{n}, n<22$ in the LGH obtained are negative. It should be noted that in this approach the importance of association effects is ignored.

The unique theory where it appeared possible to consider the formation of bound states is $2 \mathrm{D}$ classical Coulomb gas of point particles. It also can be represented by 2D $X Y$-model on the square lattice $[52,53]$ (see also [54]) with the Hamiltonian:

$$
\mathcal{H}=-\mathcal{J} \sum_{\mathbf{n}, \Delta} \cos \left(\theta_{\mathbf{n}}-\theta_{\mathbf{n}+\Delta}\right)
$$

In the continual limit it is mapped onto sin-Gordon field model [49]. Here the conductor-insulator transition occurs at small densities. The existence of the KosterlitzThouless (KT) transition $[52,53]$ may affect the $3 \mathrm{D}$ behaviour in the view of $(2+\epsilon)$-renormalization. Some numerical MC results have been interpreted from this point of view [33]. The key points of KT theory of diluted 2D Coulomb gas are: 1) the consideration of influence of the associated dipole pairs on the interaction energy of two charges through the dielectric permittivity; 2) the last is connected with the dielectric susceptibility $\chi ; 3)$ in its turn $\chi$ is determined in a self-consistent way with the polarizability of an ionic pair. Thus the equation for the dielectric permittivity is derived. This is the main point of KT-theory [55]. In such model the dissociationassociation transition (metal-insulator) takes place. The renormgroup consideration of the model [56] gives the equation for the $T_{c}$ :

$$
\beta_{c} \pi J-2-\exp \left(-\frac{\pi^{2}}{2} \beta_{c} J\right)=0 .
$$




\section{L. KULINSKII, N. P. MALOMUZH}

In this point the correlation length $\xi$ which is proportional to the size of a pair has singular behaviour:

$$
\xi=\left\{\begin{array}{cc}
\exp \left(a \tau^{-\frac{1}{2}}\right), & T>T_{c} \\
\infty, & T<T_{c}
\end{array}\right.
$$

The infinite value of correlation length in insulating phase implies the algebraic behaviour of the chargecharge correlation function:

$$
\langle\rho(0) \rho(\mathbf{r})\rangle \propto\left(\frac{a}{r}\right)^{\frac{1}{2 \pi \beta 3}}, \quad T<T_{c}
$$

It leads to the singularity in the susceptibility:

$$
\chi=\left\{\begin{array}{cc}
\xi^{2-\eta}, & T>T_{c} \\
\infty, & T<T_{c}
\end{array},\right.
$$

with $\eta=1 / 4[57]$.

There are different views on the nature of the end point of KT line. The analysis of [58] claims this point to be of usual critical type, while the extended DH model developed in [38] predicts it to be a tricritical one.

It is not quite clear also how to extend KT analysis to higher densities. It is well known that the case of two dimensions is very specific for critical phenomena because of an infinite dimension of the conformal symmetry group. Indeed, as we can see the Poisson equation (PE) for inhomogeneous isotropic dielectric media

$$
\partial(\epsilon(\mathbf{r}) \partial \phi(\mathbf{r}))=-S_{d} \rho(\mathbf{r}), \quad S_{d}=\frac{2 \pi^{d / 2}}{\Gamma(d / 2)}
$$

can be interpreted as the PE for homogeneous media, in conformally equivalent metric:

$$
d s^{2}=\epsilon(\mathbf{r}) d l^{2}
$$

where $d l^{2}=d x^{i} d x_{i}$ usual Euclidean metric. Indeed, the Laplace or Laplace-Beltrami operator for arbitrary metric $d s^{2}=g_{i k} d x^{i} d x^{k}$ is (see, e.g., [59])

$$
\Delta_{\mathrm{LB}}=\frac{1}{\sqrt{g}} \partial_{i}\left(\sqrt{g} g^{i k} \partial_{k}\right)
$$

where

$$
g=\operatorname{det}\left\|g_{i k}\right\|
$$

Comparing (25) with (23) and taking into account that the density of any quantity includes the $1 / \sqrt{g}$ factor we get that inhomogeneous PE (23) takes the form:

$$
\Delta_{\mathrm{LB}} \phi(\mathbf{r})=-S_{d} \tilde{\rho}(\mathbf{r}), \quad \tilde{\rho}=\frac{\rho}{\sqrt{g}}
$$

which is nothing but the PE for curved $D$-dimensional space with the metric tensor:

$$
g_{i k}=(\epsilon(\mathbf{r}))^{\frac{2}{\mathbf{d}-2}} \delta_{\mathbf{i k}}, \quad \mathbf{d} \neq \mathbf{2}
$$

The spaces with metric tensor $g_{i k}=f(\mathbf{r}) \delta_{i k}$ are called conformally flat, i.e. their metric tensors are proportional (the $2 \mathrm{D}$ case is the exception because any curved surface is conformally equivalent to the 2-plane). So if there exists the change of coordinates (conformal transformation) so that the Euclidean metric transforms into $g_{i k}=f(\mathbf{r}) \delta_{i k}$ the Green's function for such metrics can be obtained with the help of the Green's function for flat geometry by the change of variables. In dimensions $D \geq 3$ the conformal transformations (the so-called conformal group) are rotations, dilatations and inversions. The only inhomogeneous transformations are inversions but they lead to singular $\epsilon(\mathbf{r})=\frac{1}{|\mathbf{r}|^{2}}$. In two dimensions any complex analytical transformation of coordinates $z=(x, y) \rightarrow w=f(z)$ leads to conformally flat metric.

As is known the absence of the characteristic length scale is the reflection of the conformal invariance of the system. For two dimensional systems with the behaviour similar to the KT model (insulator-conductor) the conformal symmetry apparently forbids the spatial inhomogeneous phases. Apart from this in a $3 \mathrm{D}$ case we can expect the appearance of such phases. A typical example of such a phase is the excitonic drop phase in solid state $[60]$.

\section{Qualitative analysis of the critical behaviour of the dipole liquid}

$\mathrm{NaCl}$ is the simplest example of ionic liquid. In the solid state it is ionic crystal. Above the melting point the positions of ions become unfixed, but this liquid remains strongly dissociated. Due to thermal expansion at increasing temperature the dissociation degree diminishes and the molten salt passes to a dipole liquid. At further increasing temperature and decreasing the molten density the dissociation degree grows again and the molten salt becomes a completely ionized system. Thus, at some temperature $\left(T_{1}, T_{2}\right)$ and density $\left(n_{1}, n_{2}\right)$ intervals the molten $\mathrm{NaCl}$ can be considered as a dipole liquid. The question about the degree of ionization of IL near their critical points has been discussinged for a long time [14]. The studies of this problem were done in the early 1970ies in [61]. The van der Waals model with hard core interaction for the fluid of diatomic molecules was used. The estimations were obtained by the linear extrapolation of the density data available [62] assuming the validity of the law of rectilinear diameter. The comparison of these results with those obtained for completely ionized state near the critical point favoured the assumption about a low degree of ionization at the critical point. Further additional arguments for this assumption will be given. 
Let us consider general properties of this liquid and, in particular, its main critical parameters: the critical temperature and density. The interparticle interaction in a dipole system takes the form:

$$
U(1,2)=U_{w}(1,2)+U_{d d}(1,2)
$$

where the first term describes the van der Waals interaction between molecules and

$$
U_{d d}=\frac{1}{\epsilon r_{12}^{3}}\left(\mathbf{d}_{1} \mathbf{d}_{2}-3 \frac{\left(\mathbf{d}_{1} \mathbf{r}_{12}\right)\left(\mathbf{d}_{2} \mathbf{r}_{12}\right)}{r_{12}^{2}}\right)
$$

the proper dipole-dipole interaction, $r_{12}=\left|\mathbf{r}_{1}-\mathbf{r}_{2}\right|$ is the interparticle spacing, $i=\mathbf{r}_{i}, \mathbf{d}_{i}, \mathbf{d}_{i}=d_{0} \mathbf{n}_{i}, i=1,2$, $\epsilon$ is dielectric permittivity. Because the dipole-dipole interactions are relatively weak the angular distribution of dipole moments $\mathbf{d}_{i}$ is close to the isotropic one. More exactly we assume that the two particle distribution function $g\left(\mathbf{d}_{1}, \mathbf{d}_{2}\right)$ can be approximated by the first two terms in the expansion:

$$
g\left(\mathbf{d}_{1}, \mathbf{d}_{2}\right)=1-\beta U_{d d}(1,2)+\ldots
$$

The approximation of such a kind allows us to exclude the orientational degrees of freedom in the configurational integral with the help of the perturbation theory. In fact this procedure is equivalent to the usage of the isotropic potential

$$
\begin{aligned}
& U\left(r_{12}\right)=\langle U(1,2)\rangle=U_{w}\left(r_{12}\right)-U_{d}\left(\frac{\sigma}{r_{12}}\right)^{6} \\
& U_{d}=\frac{2}{3} \beta \frac{\ll d^{2} \gg^{2}}{\epsilon^{2} \sigma^{6}}
\end{aligned}
$$

Here $\sigma \approx a_{+}+a_{-} \approx 2 a, a_{+}$and $a_{-}$are the diameters of ions $\mathrm{Na}$ and $\mathrm{Cl}$ correspondingly and for simplicity we put $a_{+}=a_{-}$and neglect the difference in masses of the ions, $\ll \ldots \gg$ denotes the average with the internal partition function of a pair. It is easy to check that the inequality $\left|U_{w}\left(r_{12}\right)\right| \ll U_{d}\left(\frac{\sigma}{r_{12}}\right)^{6}$ takes place at all $r_{12}$. Therefore further the contribution $\left|U_{w}\right|$ will be ignored.

It is essential, that the averaging procedure restricts the applicability region of the potential (31) by interparticle spacings $\sigma \leq r_{12}$ which gives the size of the "averaged" dipole of the order $2 a$. Though the value of $\sigma$ may be slightly less than $2 a$ since rotating dipoles are not the same as hard spheres of diameter $2 a$. It is quite clear in view of scattering cross section for the hard rotating dumbells. At this level $\sigma$ should be considered as the parameter (in general temperature dependent) of the dipole-dipole potential. The procedure of its fixing in the critical point will be discussed further.

To describe the properties of the molten $\mathrm{NaCl}$ within the interval, where it can be considered as a dipole liquid, we can use the potential with hard wall:

$$
U\left(r_{12}\right)= \begin{cases}\infty & , r_{12}<\sigma \\ -U_{d}\left(\frac{\sigma}{r_{12}}\right)^{6} & , \sigma \leq r_{12}\end{cases}
$$

Such a potential leads to the van der Waals equation of state

$$
P=\frac{n_{d} k_{B} T}{1-n_{d} b}-A(T) n_{d}^{2}
$$

where

$$
A(T)=-\pi \int_{\sigma}^{\infty} U(r, T) r^{2} d r=\frac{\pi \sigma^{3}}{3} U_{d}, \quad b=\frac{2 \pi}{3} \sigma^{3}
$$

and $n_{d}$ is the pair number density. Therefore the overall density is $n=2 n_{d}$. In dimensionless form (33) and (34) read as:

$$
P^{*}=\frac{\rho^{*} T^{*}}{2-b \rho^{*}}-\frac{\tilde{A}\left(T^{*}\right)}{4} \rho^{*^{2}}
$$

and

$$
\tilde{A}\left(T^{*}\right)=\frac{2 \pi}{9 T^{*} \sigma^{3}} \ll r_{d}^{2} \gg^{2}
$$

Here all spatial parameters are given in units of $a$. The value of the parameter $\ll r_{d} \gg$ depends on the internal structure of the pair. Though by the order of magnitude $\ll r_{d} \gg \approx 1$, nevertheless from [63] it follows that the characteristic values of dipole moments correspond to $\ll r_{d} \gg<1$.

Since $\sigma$ is connected with the size of the pair we model its temperature dependence via relation:

$$
\sigma=\ll r \gg \delta
$$

where $\delta$ is the fitting parameter.

Note that $\sigma$ is temperature dependent which is assumed to be the same as that for $\ll r \gg$. The functions $\ll r^{n} \gg$ will be determined below. Note that the vapour phase, which contacts the liquid one, is the gas of dipole molecules. The van der Waals EOS is appropriate approximation for EOS for such vapour phase. Thus we can get the critical parameters of this system using the van der Waals theory of the critical point.

Equation (33) leads to the following equations for the critical temperature and density (note that $n_{d}=n / 2$, where $n$ is the total number density):

$$
T_{c}^{*}=\frac{2 \sqrt{2}}{9 \sigma^{3}} \ll r_{d}^{2} \gg, \quad \rho_{c}^{*}=\frac{1}{\pi \sigma^{3}},
$$

The estimates for these parameters are straightforward if we put $\sigma=2$, and take into account that due to small 
dipole moment of $\mathrm{NaCl} \ll r_{d}^{2} \gg=1$ (in units of $a$ ):

$$
\begin{gathered}
T_{c}^{*}=\frac{\sqrt{2}}{36} \approx 0.04, \quad \rho_{c}^{*}=\frac{1}{8 \pi} \approx 0.04, \\
P_{c}^{*}=\frac{\sqrt{2}}{1536 \pi} \approx 3 \cdot 10^{-4}, \\
Z_{c}=\frac{P_{c}^{*}}{\rho_{c}^{*} T_{c}^{*}}=\frac{3}{16} \approx 0.19
\end{gathered}
$$

which are in satisfactory agreement with the values

$$
\begin{gathered}
T_{c}^{*}=0.055, \quad \rho_{c}^{*}=0.026, \quad P_{c}^{*}=3.6 \cdot 10^{-4}, \\
Z_{c}=0.25
\end{gathered}
$$

obtained within extended Debye-Hückel-Bjerrum theory [64] augmented with ion-dipole interaction. Our value of the critical density is greater due to neglecting the dissociation of the dipole pairs.

Now we need to consider the dipole pair as itself since the parameters of potential (32) actually are the averages over the internal partition function of a pair and therefore are the temperature dependent functions.

\section{E. The dissociation of the rotating dipole liquid}

In the previous section the model of completely associated ionic liquid consisting of rotating dipoles has been introduced. Here we investigate the internal structure of the bound pair of ions. We take into account the fact that the energy of interaction of a pair should include centrifugal energy together with Coulombic potential as in standard problem of two bodies interacting via central field.

The dissociation temperature for NaCl-molten is determined by the effective potential of an ion within a rotating dipole which includes the centrifugal energy:

$$
k T_{d} \approx-U_{\mathrm{eff}}=\frac{q^{2}}{r}-\frac{L^{2}}{2 I}
$$

where $I=\mu r^{2}$ is the moment of inertia of the charge with reduced mass $\mu=\frac{m_{+} m_{-}}{m_{+}+m_{-}}=m / 2$. At such high temperatures all degrees of freedom are all in equilibrium and we can use the estimate

$$
E_{\mathrm{rot}}=\left\langle\frac{L^{2}}{2 I}\right\rangle=k T
$$

Note that the equilibrium distance between ions in a pair, which is determined by the minimum of effective potential (41) with the help of (42), is

$$
a_{e q}=\frac{1}{2 T^{*}}
$$

which is exactly the Bjerrum size of the pair $R^{B j}$ [40] (see also [64]). The choice of (43) as the size of the ionic pair is inappropriate from the physical point of view at low temperatures $T^{*} \ll 1$ [64]. It is natural that with lowering $T$ the size of a pair should become smaller tending to $a$ at $T \rightarrow 0$. That is why it was suggested to use it for $1 / T^{*} \geq 2$ only.

Let us consider this question within the picture formulated above. To be more correct, we will include the rotational energy into association constant, which is proportional to the internal partition function of the pair $[12,64]$ :

$$
K\left(T^{*}, R\right)=4 \pi \int_{a}^{R} \exp \left(-\beta U_{\mathrm{eff}}\right) r^{2} d r
$$

In $2 \mathrm{D}$ case one can put $R=\infty$ because of the logarithmic growth of the electrostatic potential and get the estimation of Berezinskii-Kosterlitz-Thouless (KT) temperature of dissociation [53]. In $3 \mathrm{D}$ case there is the problem with upper cutoff in such an approach where the association constant is identified with internal partition function of the ionic pair.

To define the size of a pair following Bjerrum we investigate the extremal points of the integrand in (44). Doing so we get two solutions:

$$
\begin{aligned}
& R_{-}\left(T^{*}\right)=\frac{1-\sqrt{1-16 T^{*} \lambda}}{4 T^{*}} \\
& R_{+}\left(T^{*}\right)=\frac{1+\sqrt{1-16 T^{*} \lambda}}{4 T^{*}}
\end{aligned}
$$

where

$$
\lambda=\frac{L^{2} / 2 I_{0}}{q^{2} / a}, I_{0}=\mu a^{2} .
$$

Here $R_{+}$is a solution of the Bjerrum type (minimum of the integrand in (44)), which as has been said above is inappropriate. $R_{-}$is another solution corresponding to the maximum of the integrand, which has quite reasonable values and correct behaviour at low $T^{*}$. It is easy to check that asymptotically for low values of the temperature $T^{*}$ the value of $K\left(T^{*}\right)$ is formed mainly by the maximum of the integrand. In addition the appropriate limiting behaviour to the hard-core contact at formal $\operatorname{limit} T^{*} \rightarrow 0$ is hold provided that $\lambda=\frac{1}{2}$. This value of $\lambda$ is in full accordance with the virial theorem [39]. All these facts confirm that we can treat the quantity $R_{-}$as the size of the pair even at 'high' temperatures $T^{*} \leq 0.1$. In addition $R_{-}$never exceeds 2 , i. e., the interparticle the 
distance when the influence of other pairs and charges on the effective potential can be neglected (see Fig. 1).

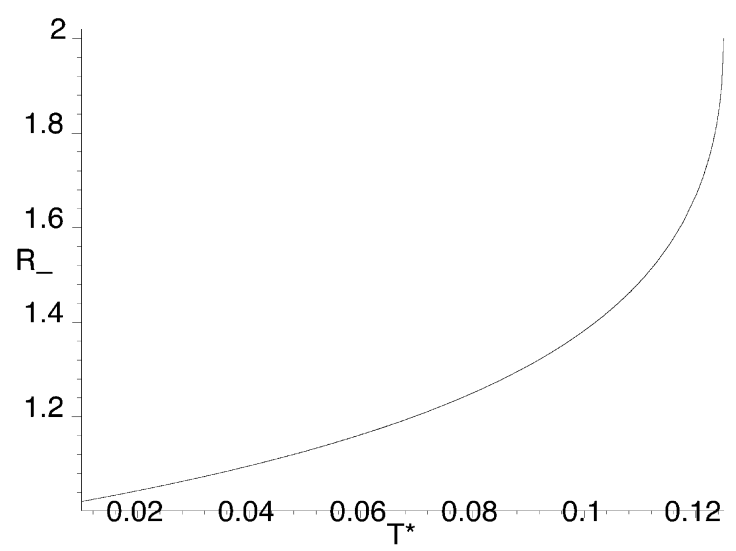

Fig. 1. The temperature dependence of $R_{-}$.

So we put $R_{-}$as the physical cutoff for (44). The existence of such length scale was noted in [64] basing on the the numerical analysis of the function $K\left(T^{*}, R\right) / K\left(T^{*}, R^{\mathrm{Bj}}\right)$, though only Coulomb potential was included in Boltzmann factor. It gives the rate at which $K\left(T^{*}, R\right)$ rises very rapidly to its plateau value. In our case we find the same behaviour of $K\left(T^{*}, R\right)$ at small temperatures, $T^{*}<0.04$ (see Fig. 2).

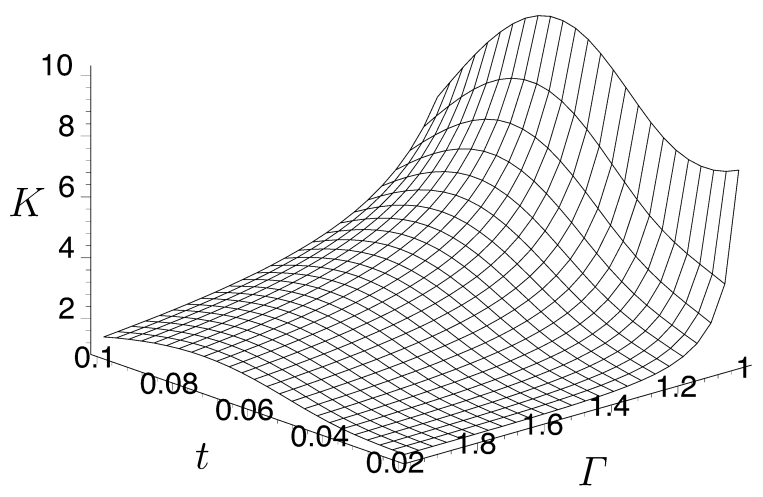

Fig. 2. The ratio $\tilde{K}=K\left(T^{*}, R_{+}\right) / K\left(T^{*}, \delta \cdot R_{-}\right)$as a function of $\delta$ and $T^{*}$.

Finally we see that there is the natural temperature interval for dipole fluid which is bound from above by the temperature

$$
T_{\mathrm{upper}}^{*} \leq \frac{1}{8}
$$

Therefore for $T<T_{\text {upper }}^{*}$ the dipole is stable in itself. Note that the existence of the temperature (46) reminds Kosterlitz-Thouless (KT) transition in a $2 \mathrm{D}$ case. In particular, the centrifugal energy introduced above plays the role analogous to the chemical potential "... required to create a pair of particles of equal and opposite charge at a distance $r_{0}$ apart ..." in Kosterlitz-Thouless model [53]. However, in contrast to $2 \mathrm{D}$ case in $3 \mathrm{D}$ case there is no any divergence in the size of the pair and therefore in its polarizability, but its derivative on the temperature has singular behaviour. In other words, the temperature derivative of the polarizability is singular but not the polarizability itself. This inference might seem as mere an artifact of introducing the upper cutoff in (44). But it should be noted that taking dielectric permittivity $\epsilon$ as the order parameter, which is directly connected with polarizability, we get exactly the divergence of its temperature derivative even in mean field approximation. This may serve as additional support for the conjecture of intensive breaking of the dipole pairs at the critical point observed in some numerical experiments [33]. Note that our estimate (46) of $T_{\text {upper }}^{*}$ perfectly corresponds to the temperature $T_{m}^{*}$ at which the maximum of the specific heat was observed in MC simulation study of the RPM performed in [33].

The existence of the interaction between dipoles and the free charges provides additional instability mechanism for their dissociation thus reducing the temperature of 'ideal' dissociation (46) because of the polarization of the dipole in the external field of dipole-dipole potential (32) and Coulombic field of free charges. The consideration given above states that there are two characteristic transition in the dipole-dipole fluid: 1) 'dipole liquiddipole gas' critical point of van der Waals type; 2) the smeared dissociation 'transition' from associated state to almost completely dissociated one. This smeared transition can be characterized by the temperature on the binodal at which the degree of dissociation is $\frac{1}{2}$.

The interaction between translational degrees of freedom of the ions is characterized by the critical temperature of the liquid-gas transition, while internal, rotational degrees of freedom are involved into dissociation of such dipole fluid. These degrees of freedom will strongly interact if the corresponding potentials will be of the same magnitude, i. e., $T_{c} \approx T_{d}$. The additional confirmation of coincidence of such transitions is the high degree of dissociation above the critical point observed in numerical experiments [33,65] and theoretical models [12], which incorporate dielectric permittivity resulting from the existence of the dipole pairs. This means that $T_{d}$ cannot be less than $T_{c}$. All said above means that in such a situation we cannot rely on (33) since appropriate EOS should incorporate all relevant interactions which lead to phase separation. In particular, the critical temperature is sensitive to the temperature dependence of the parameter $A(T)$.

The condition for the dissociation of a pair in external electrostatic field is:

$$
\langle\mathbf{d} \cdot \mathbf{E}\rangle=-\ll U_{\mathrm{eff}} \gg,
$$

where 


$$
\mathbf{d}=\mathbf{d}_{0}+\mathbf{d}_{\text {ind }}
$$

is the dipole moment, which consists of proper and induced dipole moments. Obviously,

$$
\left\langle\mathbf{d}_{0} \cdot \mathbf{E}\right\rangle=0, \quad\left\langle\mathbf{d}_{\text {ind }} \cdot \mathbf{E}\right\rangle=\chi\left\langle\mathbf{E}^{2}\right\rangle
$$

Here $\chi$ is the polarizability of a pair. The averaging over the thermal equilibrium gives:

$$
\left\langle\mathbf{E}^{2}\right\rangle=\frac{3 k_{B} T}{\varkappa V_{\mathrm{ph}}},
$$

where $\varkappa$ is the dielectric susceptibility of the medium and $V_{\mathrm{ph}}$ is the characteristic volume. It is connected with the polarizability of the cavity. According to the definition:

$$
\varkappa=\frac{\epsilon-1}{4 \pi}=\frac{1}{2} \chi \rho^{*}
$$

So we get the equation for the temperature in a dimensionless form:

$$
\frac{6}{\rho^{*} V_{\mathrm{ph}}} T^{*}=-\ll U_{\mathrm{eff}} \gg
$$

Within the proposed approach we put $V_{\mathrm{ph}}=\frac{4 \pi}{3} l_{c}^{3}$ where $l_{c}$ is the radius of first coordination sphere. This is the minimal volume for which the conception of continuity of the medium can be applied. By the order of magnitude $l_{c} \approx 1.5 \mathrm{a}$. The solution of (52) gives the dependence $T^{*}(\delta)$. In order to fix the value of $\delta$ in the critical point which determine the size of the pair we should equate $T^{*}(\delta)$ and $T_{c}^{*}(\delta)$ obtained above. This way we get:

$$
\begin{aligned}
& T_{c}^{*}=0.048, \quad \rho_{c}^{*}=0.054, \quad P_{c}^{*}=4.8 \cdot 10^{-4} \\
& \sigma=1.8, \quad Z_{c}=0.19
\end{aligned}
$$

which are close to those obtained above (39). In notations of [64], $\sigma=2 a_{2}$. In this work the estimate for the parameter $a_{2}$ from simple geometric considerations was given: $0.825 \leq a_{2} \leq 1.565$. Thus our estimate is in this interval. From the results obtained above we can infer that the dipole fluid of rotating dipoles in the vicinity of its liquid-gas critical point is about to dissociate. Sure our consideration is incomplete since it does not take into account the existence of free charges.

Finally we estimate the Ginzburg number by the formula used for the molecular liquids [39]:

$$
G i=\left(\frac{r_{0}}{\xi_{0}}\right)^{6}
$$

where $r_{0}=\ll r \gg \approx a$ is the interparticle spacing within ionic pair and $\xi_{0}$ is the amplitude of the correlation length for density fluctuations. Since the density fluc- tuations are connected with the ones for dipole pairs we put it to be equal $\xi \geq \sigma$. Using the parameters of the critical point found in (53) we get the estimate:

$$
G i \leq 0.04
$$

\section{Remarks}

The ionic and dipole liquids form two natural approximations to describe the critical properties of the systems similar to the molten $\mathrm{NaCl}$. In our paper we have estimated the main critical parameters for liquid with hard dipole as well as consider the influence of the effects arising due to softness of a dipole molecule. In particular the latter is very important to describe the dielectric properties of a system near the critical point. Besides, the variation of molecule parameters due to the rotations allows us to determine the equilibrium size of a ionic pair.

It is not excluded that the quantum corrections to internal states of the dipole pairs will also slightly change the estimates. In particular the temperature dependence of the vibrational contributions to the heat capacity can also be studied. The following step is to construct the equation of state for small 'soft' dipole molecules and to take into account the dissociation process with the help of perturbation theory. The combination of such an approach with that developed in [17] on the basis of ionic liquid allows to narrow the region of the most probable values for the critical parameters.

Our estimate for the critical temperature correlates with the known analytical results. Note that most of the analytical approaches based on EOS for low density Coulombic system (DH, MSA, etc.), where the dissociation is taken into account perturbatively.

Within the dipole liquid approach we have obtained the estimate for the Ginzburg temperature and have shown that it less than the one for a simple liquid by the factor $10^{-2} \div 10^{-1}$. The approximation of the dipole liquid allows us to analyze in the evident form the contribution of the polarizational effects [17]. One can show that the latter lead to a further considerable decrease of the Ginzburg temperature.

Note also the possibility for the appearance of new inhomogeneous phase near the critical point of IL. Since the dissociation temperature $T_{d}$ is near $T_{c}$, the system can desintegrate on the regions with the essentially different values of the degree of ionization $\Delta$ : the drops of ionic and dipole liquids. As a consequence the region with the Ising-like behaviour cannot be reached. This scenario calls for a very careful investigation. These and other questions will be studied further.

\section{POLARIZATIONAL INTERACTIONS}

The definitive role for the critical behaviour of systems with CI belongs to the charge-order parameter interactions. This question was discussed in [66] concerned to 
the structure of electrolyte solutions near their critical point and super-ionic crystals $[67,68]$. So in [66] it was assumed that the Hamiltonian of an electrolyte has the structure:

$$
H=H_{\mathrm{LG}}+H_{\mathrm{ch}}+H_{\mathrm{int}},
$$

where $H_{\mathrm{LG}}$ is the standard LGH,

$$
H_{\mathrm{ch}}=\frac{1}{2} \sum_{\alpha, \beta} \int d \mathbf{r} \int d \mathbf{r}^{\prime} \frac{\delta n_{\alpha}(\mathbf{r}) \delta n_{\beta}\left(\mathbf{r}^{\prime}\right)}{\epsilon_{0}\left|\mathbf{r}-\mathbf{r}^{\prime}\right|}
$$

the Hamiltonian of interionic interaction, and

$$
H_{\mathrm{int}}=\sum_{\alpha} g_{\alpha} \int \eta(\mathbf{r}) n_{\alpha}(\mathbf{r}) d \mathbf{r}
$$

the Hamiltonian of charge-order parameter interaction. In fact, the last takes into account only the short-range interaction between the order parameter and ions in a system and does not describe the electrostatic effects.

On the basis of the Hamiltonian (56) in [66] the new inhomogeneous phase in the vicinity of the critical point was predicted. However, its existence was not confirmed in further experiments. It shows explicitly the incorrectness of the assumptions about 1) contact character of charge-order parameter interaction and 2) interionic in- teraction in the form (58) corresponding to constant value of the dielectric permittivity.

The analogous contact character of the chargedeformation tensor interaction is postulated in works $[67,68]$, devoted to the phase transitions in super-ionic crystals. Here we note that the dielectric permittivity of crystal changes due to its deformation. Therefore the electric potential $\phi$ inside system will be definitive functional of the deformation tensor. As the result the energy of Coulombic interaction:

$$
f_{\mathrm{ch}}=\frac{1}{2} \int \rho \phi d V
$$

includes the charge-deformation tensor interaction by itself.

\section{A. General theory of polarizational interactions}

The free energy of the system with the charge fluctuations near its critical point can be represented in the form:

$$
F[\eta(\mathbf{r}), \phi(\mathbf{r})]=F_{\mathrm{reg}}+F_{\mathrm{LG}}\left[\eta(\mathbf{r}]+C_{\mathrm{el}}[\eta(\mathbf{r}), \phi(\mathbf{r})],\right.
$$

where $F_{r}$ is the regular part, $F_{\mathrm{LG}}$ is the LandauGinzburg functional:

$$
F_{\mathrm{LG}}[\eta(\mathbf{r})]=\int d V\left(\frac{c}{2}(\nabla \eta(\mathbf{r}))^{2}+a_{1} \eta(\mathbf{r})+\frac{a_{2}}{2} \eta(\mathbf{r})^{2}+\frac{a_{4}}{4} \eta(\mathbf{r})^{4}\right)
$$

depending on the order parameter $\eta(\mathbf{r})$ and

$$
C_{e l}[\eta(\mathbf{r}), \phi(\mathbf{r})]=\frac{1}{2} \int d V \rho(\mathbf{r}) \phi(\mathbf{r})=\frac{1}{2} \iint d \mathbf{r} d \mathbf{r}^{\prime} G\left[\mathbf{r}_{1}, \mathbf{r}_{2} \mid \epsilon\left(\mathbf{r}^{\prime}\right)\right] \delta \rho\left(\mathbf{r}_{1}\right) \delta \rho\left(\mathbf{r}_{2}\right)
$$

is the contribution of the charged subsystem, where $G\left[\mathbf{r}_{1}, \mathbf{r}_{2} \mid \delta \epsilon\left(\mathbf{r}^{\prime}\right)\right]$ is the Green's functional for the inhomogeneous medium. In fact the characteristic time for the charge fluctuations is essentially less then that for the order parameter. Therefore, to describe the critical behaviour of the system we should average expression (61) over the charge fluctuations. Then the fluctuational deviations of the free energy from its regular part takes the form:

$$
\delta F=F_{\mathrm{LG}}[\eta(\mathbf{r})]+F_{\mathrm{pol}}[\eta(\mathbf{r})]
$$

where

$$
F_{\mathrm{pol}}[\eta(\mathbf{r})]=\left\langle C_{\mathrm{el}}[\eta(\mathbf{r}), \phi(\mathbf{r})]\right\rangle
$$

Here we assume that the fluctuations of the dielectric permittivity caused mainly the fluctuations of the total number density of the particles. In the local approximation we can write:

$$
\epsilon(\mathbf{r})=\epsilon_{c}\left(1+\lambda_{1} \eta(\mathbf{r})+\lambda_{2} \eta^{2}(\mathbf{r})+\ldots\right)
$$

where $\epsilon_{c}$ is the value of the dielectric permittivity in the critical point and

$$
\lambda_{k}=\left.\frac{n_{c}^{k}}{\epsilon_{c}} \frac{\partial^{k} \epsilon}{\partial n^{k}}\right|_{n=n_{c}} .
$$




\section{L. KULINSKII, N. P. MALOMUZH}

By the definition we will call $F_{e l}$ the polarizational contribution to the free energy of the system.

The deviation of free energy for weakly nonequilibrium state near the critical point from (59) is:

$$
\delta F[\eta(\mathbf{r}), \phi(\mathbf{r})]=\delta C_{e l}[\eta(\mathbf{r}), \phi(\mathbf{r})]+\delta F_{L G}[\eta(\mathbf{r})]
$$

Therefore,

$$
\begin{aligned}
& \delta C_{e l}[\eta(\mathbf{r}), \phi(\mathbf{r})]=-\int d V \delta \epsilon(\mathbf{r}) \frac{(\nabla \phi)^{2}}{8 \pi} \\
& +\int d V \delta \rho(\mathbf{r}) \phi(\mathbf{r}) .
\end{aligned}
$$

We also use the global electroneutrality condition

$$
Q[\rho]=\int \delta \rho(\mathbf{r}) d V=0
$$

To get simpler form of the equations we use the linear approximation for the deviation of the dielectric permittivity:

$$
\delta \epsilon=b \eta(\mathbf{r})
$$

where $b=\lambda_{1} \epsilon_{c}$ is some function of temperature and chemical potential. Its specific form depends on the choice of an order parameter $\eta$.

In this case (68) can be derived as the linear approximation using any model for density dependence of dielectric permittivity. In the case when the density fluctuations include elastic component, e.g., for solid electrolytes [67,68], (68) should include the deformation field component of the density:

$$
\delta \epsilon=-\mathbf{u} \nabla \epsilon-n \frac{\partial \epsilon}{\partial n} \operatorname{div} \mathbf{u}
$$

Next step we make is to connect the density of the order parameter field $\eta(\mathbf{r})$ with the charge density $\delta \rho(\mathbf{r})$. Assuming that the linear approximation is valid we can write:

$$
\delta \rho(\mathbf{r})=\delta \rho_{h}+\gamma \eta(\mathbf{r})
$$

where $\delta \rho_{h}$ is the charge fluctuations for the homogeneous state. Substituting (68) and (70) in (66) for (65) we get:

$$
\begin{aligned}
& \delta F[\eta(\mathbf{r}), \phi(\mathbf{r})]=\delta F_{\mathrm{LG}} \\
& -\frac{1}{2} \int d V \eta(\mathbf{r})\left(b \frac{(\nabla \phi)^{2}}{4 \pi}+2 \gamma \phi(\mathbf{r})\right)+\int \delta \rho_{h} \phi(\mathbf{r}) d V
\end{aligned}
$$

Now the basic relation between the fields $\eta(\mathbf{r})$ and $\phi(\mathbf{r})$ can be derived by standard minimization procedure for the functional (59) with condition (67). Here the variations of the fields $\phi(\mathbf{r})$ and $\eta(\mathbf{r})$ are independent and therefore:

$$
\begin{aligned}
& \frac{\delta}{\delta \eta(\mathbf{r})}(F[\eta(\mathbf{r}), \phi(\mathbf{r})]-\lambda Q[\delta \rho(\mathbf{r}), \phi(\mathbf{r})])=0 \\
& \frac{\delta}{\delta \phi(\mathbf{r})}(F[\eta(\mathbf{r}), \phi(\mathbf{r})]-\lambda Q[\delta \rho(\mathbf{r}), \phi(\mathbf{r})])=0 .
\end{aligned}
$$

It yields

$$
\begin{aligned}
& -c \Delta \eta(\mathbf{r})+a_{2} \eta(\mathbf{r})+a_{4} \eta^{3}(\mathbf{r}) \\
& -\frac{1}{2}\left(b \frac{(\nabla \phi)^{2}}{4 \pi}+2 \gamma \phi(\mathbf{r})\right)=0, \\
& b \nabla(\eta(\mathbf{r}) \nabla \phi)+4 \pi \gamma \eta(\mathbf{r})+4 \pi \delta \rho_{h}=0
\end{aligned}
$$

where we put $\lambda \gamma=-a_{1}$ because of asymptotic conditions

$$
\phi(\mathbf{r}), \eta(\mathbf{r}) \rightarrow 0, \text { if } \mathbf{r} \rightarrow \infty
$$

It is clear that for large scales $\gamma$ is very small. Thus the Green's function satisfies the inhomogeneous Poisson equation:

$$
\nabla\left(\left(\epsilon_{c}+\delta \epsilon(\mathbf{r})\right) \nabla G\left(\mathbf{r}, \mathbf{r}^{\prime}\right)\right)=-4 \pi \delta\left(\mathbf{r}-\mathbf{r}^{\prime}\right)
$$

In local approaximation the Green's function is:

$$
G\left(\mathbf{r}, \mathbf{r}^{\prime}\right)=\frac{1}{\epsilon_{c}\left(1+\delta \tilde{\epsilon}\left(\mathbf{r}-\mathbf{r}^{\prime}\right)\right)} \frac{1}{\left|\mathbf{r}-\mathbf{r}^{\prime}\right|}
$$

Substituting (75) to (65) we obtain

$$
F_{\mathrm{pol}}[\eta]=\frac{1}{2} \int d \mathbf{r} \int d \mathbf{r}^{\prime} \frac{\left\langle\delta \rho(\mathbf{r}) \delta \rho\left(\mathbf{r}^{\prime}\right)\right\rangle}{\epsilon_{c}\left|\mathbf{r}-\mathbf{r}^{\prime}\right|}\left(\frac{1}{1+\delta \tilde{\epsilon}(\mathbf{r})}-1\right)
$$

The polarizational energy for the spatially homogeneous state is equal to:

$$
F_{h}=\frac{1}{2} \int d \mathbf{r} \int d \mathbf{r}^{\prime} \frac{\left\langle\delta \rho(\mathbf{r}) \delta \rho\left(\mathbf{r}^{\prime}\right)\right\rangle}{\epsilon_{c}\left|\mathbf{r}-\mathbf{r}^{\prime}\right|}
$$

and is excluded from (65). In the simplest limiting Debye-Hückel approximation:

$$
\beta F_{h}^{(e q)}=-\frac{1}{12 \pi} \Gamma_{c}^{3}
$$

where

$$
\Gamma=\frac{a}{r_{s}}
$$


is the inverse screening length amplitude. The inclusion of hard core effects leads to

$$
F_{h}-F_{i d}=-\frac{1}{4 \pi}\left(\ln (1+\Gamma)-\Gamma+\Gamma^{2} / 2\right)
$$

in DH model. Other approach based on the mean spherical approximation for the correlation function gives:

$$
F_{h}-F_{\mathrm{id}}=-\frac{1}{12 \pi}\left(2+6 \Gamma+3 \Gamma^{2}-2(1+2 \Gamma)^{3 / 2}\right) .
$$

Therefore we get:

$$
F_{\mathrm{pol}}[\eta]=F_{h} \int d V\left(\frac{1}{1+\delta \tilde{\epsilon}(\mathbf{r})}-1\right)
$$

Thus (81) can be represented in the form:

$$
F_{\mathrm{pol}}=\int d \mathbf{r} \sum_{n=1}^{\infty} \frac{1}{n} a_{n}^{(\mathrm{pol})} \eta^{n}(\mathbf{r})
$$

where

$$
\begin{gathered}
a_{1}^{(\mathrm{pol})}=-\lambda_{1}, \quad a_{2}^{(\mathrm{pol})}=-\lambda_{2}+\lambda_{1}^{2}, \\
a_{3}^{(\mathrm{pol})}=-\lambda_{3}+2 \lambda_{1} \lambda_{2}-\lambda_{1}^{3}, \\
a_{4}^{(\mathrm{pol})}=-\lambda_{4}+2 \lambda_{3} \lambda_{1}+\lambda_{2}^{2}-3 \lambda_{2} \lambda_{1}^{2}+\lambda_{1}^{4},
\end{gathered}
$$

In fact after neglecting the terms of order $n>4$ this gives an addition to the initial LGH of the system (60).

Note that as far as expression (81) which accounts for polarizational effects used the polarizational contribution to $a_{4}$ is negative. It follows from the facts that: a) the dielectrical permittivity is monotonic function of the density and b) the Coulombic potential is convex function of the dielectric permittivity c) the excess free energy for ionic system is negative. It becomes quite clear if we choose $\eta=\frac{\epsilon-\epsilon_{c}}{\epsilon_{c}}$ as the order parameter. The including of (81) to the effective LGH leads to the diminishing of the value of $a_{4}$ thus reducing the Ginzburg number.

The results obtained above serve as the background for analysis the Ginzburg number in molten salt.

\section{B. Effective Landau-Ginzburg Hamiltonian for ionic liquids}

According to [18] the effective LGH of molten salt $\mathrm{NaCl}$ takes the structure:

$$
\beta H_{\mathrm{eff}}[\eta(\mathbf{r})]=\int d \mathbf{r}\left(\frac{b_{2}}{2}(\nabla \eta(\mathbf{r}))^{2}+\sum_{m=1}^{4} \frac{a_{m}}{m} \eta^{m}(\mathbf{r})\right),
$$

where

$$
a_{m}=a_{m}^{(0)}+a_{m}^{(\mathrm{pol})}
$$

and

$$
b_{2}=b_{2}^{(0)}
$$

The explicit expressions for $a_{m}^{(\mathrm{pol})}$ follow from the consideration of polarizational interactions described in previous section.

The coefficients $a_{2}^{(0)}$ and $a_{4}^{(0)}$ are connected with the derivatives of pressure with respect to density by the relations:

$$
a_{2}^{(0)}=\left.\frac{\partial P}{\partial n}\right|_{T}, \quad a_{4}^{(0)}=\left.\frac{\partial^{3} P}{\partial n^{3}}\right|_{T},
$$

in which $P$ includes the contributions of both short-range repulsive and Coulombic interactions. Almost all equations of state for the RPM lead to very small values of the coefficients $a_{i}^{(0)}$ compared with those for molecular fluids at least by an order of magnitude $[69,70]$.

$$
\frac{a_{2}^{(\mathrm{RPM})}}{a_{2}^{(\mathrm{LJ})}}=0.01 \div 0.1, \quad \frac{a_{4}^{(\mathrm{RPM})}}{a_{4}^{(\mathrm{LJ})}}=0.001 \div 0.01 .
$$

The only exception is Debye-Hückel-Bjerrum (DHBj) model where the values of these coefficients are of the same order as for molecular fluid. To calculate $a_{m}^{(\mathrm{pol})}$ we use the formulas $(83),(84)$ with coefficients $\lambda_{k}$, determined with the help of the canonical form for dielectric permittivity:

$$
\frac{\epsilon-1}{\epsilon+2}=\lambda(1+\eta)
$$

where

$$
\lambda=\frac{4 \pi}{3} \alpha_{\mathrm{eff}} \rho_{c}^{*}
$$

and $\rho^{*}=n a^{3}$. Here $n$ is the overall number density. In accordance with $(90)$ the parameter $\lambda$ satisfies the inequality $\lambda<1$. The value of $\epsilon$ does not exceed 1.2 (in vapour phase) $[12,64]$.

The effective polarizability $\alpha_{\text {eff }}$ is mainly formed by the associated ionic pairs:

$$
\alpha_{\text {eff }}=\frac{1-\Delta}{6} \delta^{2} \frac{1}{T^{*}},
$$




\section{L. KULINSKII, N. P. MALOMUZH}

where

$$
\Delta=\frac{n_{+}}{n_{0}}=1-\frac{n_{\text {dim }}}{n_{0}}
$$

is the degree of dissociation of the system defined above (16), $n_{0}$ is the number density of the ions of particular type, i.e., $n=2 n_{0}, T^{*}$ is the dimensionless temperature $T^{*}=\frac{k_{B} T}{e^{2} / a}$ and $\delta=l / a$ is the dimensionless size of an ionic pair ( $d_{0}=e l$ being its dipole moment) and we put $Z_{+}=Z_{-}=1$ for simplicity. The condition $\lambda<1$ holds for all cases since $\frac{\rho_{c}^{*}}{T_{*}^{*}}$ sufficiently small. In addition if $\frac{9 T_{c}^{*}}{2 \pi \delta^{2} \rho_{c}^{*}}<1$ we can get the low estimate for $\Delta$ at the critical point:

$$
1-\frac{9 T_{c}^{*}}{2 \pi \delta^{2} \rho_{c}^{*}}<\Delta_{c},
$$

This estimate is natural for 3D Coulombic systems which apparently cannot undergo Kosterlitz-Thouless (KT) transition [33], where $\Delta=0$ with $r_{s}$ divergence. Thus the model (90) is acceptable from this point of view even for the critical point located at 'high density' $\left(\rho_{c}^{*} / T_{c}^{*}>1\right)$. Note that as $\delta$ grows the degree of dissociation $\Delta_{c}$ at the critical point becomes closer to 1 , which is pretty natural from the physical point of view.

Further analysis of the polarizational induced terms into LGH-coefficients is sensible with respect to the coordinates $\left(\rho_{c}^{*}, T_{c}^{*}\right)$ of the critical point. To estimate $\rho_{c}$ and $T_{c}^{*}$ different models for the EOS: DH, MSA, DH with account of dimerization (Bjerrum approach) and hard core interactions etc. were used (see $[6,69,70]$ ). There are also the computer simulations of the phase diagram $[32,33]$. The values of parameter $\frac{\rho_{c}^{*}}{T_{c}^{*}}$ obtained with the help of analytical methods are small,

$$
0.5<\frac{\rho_{c}^{*}}{T_{c}^{*}}<1
$$

('low critical density' case). Unlike these analytical estimates recent computer calculations give the values $1<\frac{\rho_{c}^{*}}{T_{c}^{*}}<2$ [32]. Basing on the estimate (94) one can see that both these cases are consistent $(\lambda<1)$ with the model. Note that model EOS like MSA with different corrections [12] and numerical MC calculations [33,32] also show high degree of dissociation near critical point.

\section{The effective Landau-Ginzburg Hamiltonian for electrolyte solutions}

The electrolyte solution near its vapour-liquid critical point is characterized by two independent scales: a) the screening length $r_{s c r}$, which in Debye approximation is:

$$
r_{\mathrm{scr}} \approx r_{\mathrm{D}}=\frac{1}{\Gamma}
$$

and b) the correlation length $r_{c}$ for density fluctuations in a solvent:

$$
r_{c}=r_{0} \tau^{-\nu}, \quad \nu=\left\{\begin{array}{l}
0.5 \quad \text { classical region } \\
0.63 \text { fluctuation region }
\end{array}\right.
$$

The critical behaviour of a solution depends on the interplay of these scales. For experiments where

$$
r_{\mathrm{scr}} \gg r_{c}
$$

the interionic electric field acts as the additional pressure which changes the position of the critical point. The character of fluctuations of an order parameter for the system does not change and the range for crossover of critical exponents is determined by the Ginzburg number for a solvent. In a more important second case when

$$
r_{\mathrm{scr}}<(\ll) r_{c}
$$

the electric field of charge fluctuations polarizes a solvent and as a result leads to variation of interaction constants in the LGH and also to appearance of additional terms in it.

This consideration based on the important fact that the screening length remains finite at an approaching the liquid-gas critical point. Such a conclusion is a direct consequence of the isomorphism principle for the critical phenomena in multicomponent mixtures and simple liquids. In accordance with it the only extensive variable of state is strongly fluctuating in the vicinity of the critical point. The level of fluctuations for others, which orthogonal to it is bounded. 'Switching on' the Coulombic interaction for electroneutral system additionally suppresses the long range fluctuations of the variable connected with the charge fluctuations.

The lower bound for the concentration range where Debye screening is not destroyed by the thermal fluctuations is

$$
x_{\min }=v\left(\frac{\epsilon k_{B} T}{q^{2}}\right)^{3},
$$

where $v$ is the volume per molecule of a solvent.

Because of polarization of a solvent the local electrostatic energy is fluctuating on the scales of densitydensity correlation length. The account of this contribution renormalizes the interaction constants in the LGH. To construct the corresponding LGH we will use the interrelation between fluctuations of density and dielectric permittivity,

$$
\epsilon \rightarrow \epsilon(\mathbf{r})=\epsilon\left(1+\lambda_{1} \eta(\mathbf{r})+\lambda_{2} \eta^{2}(\mathbf{r})+\ldots\right)
$$

$$
\lambda_{k}=\left.\frac{\rho_{c}^{k}}{\epsilon\left(\rho_{c}\right)} \frac{\partial^{k} \epsilon}{\partial \rho^{k}}\right|_{\rho=\rho_{c}}, \quad \eta=\rho / \rho_{c}-1
$$


Here $\epsilon$ is the equilibrium value for dielectric permittivity. We can obtain the effective LGH as follows. For homogeneous system the density of energy is a sum of nonCoulombic contribution $h_{\mathrm{nC}}$ and that for Coulombic subsystem $h_{\mathrm{C}}$. Far away from the critical point the latter has the structure

$$
h_{\mathrm{C}}=-\frac{1}{8 \pi} \Gamma_{c}^{3} \frac{1}{\left(\epsilon / \epsilon_{c}\right)^{3 / 2}},
$$

and the index " $c$ " indicate that the values are taken in the critical point. To take into account the large scale inhomogeneity of the system, we assume that the condition

$$
r_{c}>r_{\mathrm{scr}}
$$

is fulfilled, where $r_{c}$ is the correlation length for the fluctuations of an order parameter. We can use the analogous expressions for $h_{\mathrm{nC}}$ and $h_{\mathrm{C}}$ in which local values of $\epsilon$ and $n$

$$
\begin{aligned}
n & \rightarrow n(1+\tilde{\eta}(\mathbf{r})) \\
\epsilon & \rightarrow \epsilon(1+\tilde{\epsilon}(\mathbf{r}))
\end{aligned}
$$

should be substituted. As a result the fluctuational contribution of Coulombic subsystem into the energy of the system equals

$$
\beta h_{\mathrm{C}}(\mathbf{x})=-\frac{\Gamma_{c}^{3}}{8 \pi}\left[\frac{1}{(1+\tilde{\epsilon}(\mathbf{x}))^{\frac{3}{2}}}-1\right]
$$

The formal derivation of the quasilocal approximation (103) from the microscopic point of view is given in [17]. Adding the quasilocal term

$$
\beta h^{(\mathrm{ql})}=\frac{b^{2}}{2}(\nabla \eta(\mathbf{r}))^{2}
$$

we assume that the value of $b$ can be evaluated with the help of results $[69,36]$.

Using the dimensionless form of coefficients of the LGH the formulas (102) and (103) yield the following:

$$
\beta H_{\mathrm{eff}}[\eta(\mathbf{x})]=\int d \mathbf{x}\left(\frac{b}{2}(\nabla \eta(\mathbf{x}))^{2}+\sum_{m=1}^{\infty} \frac{a_{m}}{m} \eta^{m}(\mathbf{x})\right)
$$

where

$$
a_{m}=a_{m}^{(0)}+a_{m}^{(e l)} x^{3 / 2}, m \geq 1, \quad a_{m}^{(0)}=0, \quad m \geq 5,
$$

$$
b=\left(c_{0}+c\right) \sigma
$$

and according to Eq. (1)

$$
a_{1}^{(0)}=a_{3}^{(0)}=0
$$

Here $\sigma$ is the diameter of a molecule in a solvent. The values of $a_{m}^{(e l)}$ are determined from Eq. (103) and is equal to

$$
a_{m}^{(e l)}=\frac{(-1)^{m+1}}{16 \pi} \frac{(2 m+1) ! !}{(2 m-2) ! !}\left(\Gamma_{*} \sigma\right)^{3}, \quad \Gamma_{*}=\frac{\Gamma}{\sqrt{x}}
$$

The calculation of the LGH without taking into account polarization effects was done in $[69,70]$ basing on different models. The Ginzburg criterion obtained did not show the existence of the crossover $(G i \simeq 10)$.

Now it is desirable to reduce the initial Hamiltonian of an electrolyte (105) in the vicinity of the critical point $\left(r_{\mathrm{D}} \ll r_{c}\right)$ to the Landau-Ginzburg form. Usually such a reduction is performed by omitting all local terms $\sim \eta^{n}, n \geq 5$. However, this step is connected with the loss of important information about corrections to the leading asymptotic terms [71]. A more suitable way is connected with attraction of ideas of the Catastrophe Theory [72] within the framework of the so-called canonical formalism [71]. Its central conception is the canonical transformation of the order parameter:

$$
\eta \rightarrow \phi=\eta+\frac{1}{2} \gamma_{2} \eta^{2}+\frac{1}{3} \gamma_{3} \eta^{3}+\ldots \equiv \hat{C} \eta
$$

which reduce the local Hamiltonian of the system

$$
\beta H_{\mathrm{eff}}[\eta(\mathbf{x})]=\int d \mathbf{x}\left(\sum_{m=1}^{\infty} \frac{a_{m}}{m} \eta^{m}(\mathbf{x})\right)
$$

near the critical point to the canonical form, which is an analogue of that used in the Catastrophe Theory [74]:

$$
\begin{aligned}
& \beta H_{\mathrm{eff}}[\eta(\mathbf{x})]=\int d \mathbf{x} \\
& \times\left(-h_{\phi} \phi(\mathbf{x})+\frac{1}{2} A_{2} \phi(\mathbf{x})^{2}+\frac{1}{4} A_{4} \phi(\mathbf{x})^{4}\right),
\end{aligned}
$$

The canonical transformation of the order parameter plays very important role establishing the isomorphism between Ising model and liquids. Besides, the explicit expressions for the generalized external field $h_{\phi}$ and 'temperature' $A_{2}$ are obtained. The coexistence curve of liquids in new variables $\left(h_{\phi}, A_{2}\right)$ is symmetric with respect to transformation $h_{\phi} \rightarrow-h_{\phi}$ similarly to Ising model. Its asymmetry appears only if initial (noncanonical) variables are used. 
The combination of the canonical transformation with standard RG procedure allows to get new version of RG theory $[75,76]$.

\section{Canonical form for the effective Hamiltonian}

As has been shown above the Hamiltonian of the system has no Ising like form. It is essential that it includes two terms: the local $H_{\mathrm{loc}}$, i. e., k-independent, and quasilocal $H_{\mathrm{ql}}$, i.e., k-dependent. Following [73] we will show that the local part of the Hamiltonian can be exactly reduced to the canonical form $H_{\text {can }}(\eta)$ which in case of the 2-nd order phase transition coincides with classical Landau-Ginzburg functional. For convenience we include $\beta$ into the Hamiltonian. In a case of simple liquids, for example, the coefficients $a_{n}$ are definite functions of the chemical potential $\mu$ and the temperature $T$ if the nontrivial reference system is used [36]. Further, we consider only a local part of the Hamiltonian. Due to locality, for every point we can write:

$$
\phi(\mathbf{r})=F(\eta(\mathbf{r}))
$$

where the function $F(x)$ is smooth and invertible, and besides it satisfies the condition $F(0)=0$. Then for the integrand in the partition function of the system we can write

$$
\exp \left(-\mathrm{h}_{\mathrm{loc}}^{(\mathrm{can})}(\eta)\right)=\int \delta(\phi-F(\eta)) \exp \left(-\mathrm{h}_{\mathrm{loc}}^{(\text {can })}(\phi)\right) d \phi
$$

$$
\mathrm{h}_{\mathrm{loc}}^{(\mathrm{can})}(\eta)=A_{1} \eta+\frac{A_{2}}{2} \eta^{2}+\frac{A_{4}}{4} \eta^{4} .
$$

The implicit form for the canonical transformation of the order parameter $\phi$ is as follows:

$$
\int_{0}^{\eta} \exp \left(-\mathrm{h}_{\mathrm{loc}}^{(\text {(can) }}(z)\right) d z=\int_{0}^{\phi} \exp \left(-\mathrm{h}_{\mathrm{loc}}(z)\right) d z
$$

which means that the corresponding (local) Gibbsian measures coincide. The coefficients $A_{k}$ of the canonical form are determined as functions of the parameters of the initial local Hamiltonian by implying the condition that the ranges for the variables $\phi$ and $\eta$ are the same:

$$
\begin{aligned}
& \int_{0}^{+\infty} \exp \left(-\mathrm{h}_{\mathrm{loc}}^{(\text {can })}(\eta)\right) d \eta=\int_{0}^{+\infty} \exp \left(-\mathrm{h}_{\mathrm{loc}}(\phi)\right) d \phi, \\
& \int_{-\infty}^{0} \exp \left(-\mathrm{h}_{\mathrm{loc}}^{(\text {can })}(\eta)\right) d \eta=\int_{-\infty}^{0} \exp \left(-\mathrm{h}_{\mathrm{loc}}(\phi)\right) d \phi .
\end{aligned}
$$

One can show that the transformation (111) defined by (114) is analytical provided that the local part of the effective Hamiltonian is analitical too.

Moreover, since (111) is nothing but the redefining of the order parameter, the loci for the critical point both for initial and canonical Hamiltonians must coincide:

$$
\begin{gathered}
A_{1}(P, T)=0, \quad A_{2}(P, T)=0 \quad \Leftrightarrow \quad a_{1}(P, T)=0, \\
a_{2}(P, T)=0 .
\end{gathered}
$$

Sure it is implied that $a_{3}(P, T)=0$ also because of the stability condition. The constraint (116) fixes the value of $A_{4}>0$ :

$$
\begin{aligned}
& \int_{-\infty}^{+\infty} \exp \left(-\frac{A_{4}}{4} \eta^{4}\right) d \eta \\
& =\int_{-\infty}^{+\infty} \exp \left(-\mathrm{h}_{\mathrm{loc}}\left(\phi ; a_{1}=0, a_{2}=0\right)\right) d \phi .
\end{aligned}
$$

This gives

$$
A_{4}=\frac{\pi^{4}}{\left(\Gamma\left(\frac{3}{4}\right) \int_{-\infty}^{+\infty} \exp \left(-\mathrm{h}_{\mathrm{l} \perp c}\left(\phi ; a_{1}=0, a_{2}=0\right)\right) d \phi\right)^{4}}
$$

Note that the functional dependence of the canonical coefficients $A_{i}, i=1,2,4$ on the parameters $a_{i}$ is determined by the form of the initial effective Hamiltonian.
Besides, since $A_{i}$ are the coefficients of the LGH they may be used as the approximates for the renormalized fields of the LGH in the vicinity of the critical point. 
Note also that in such a way the problem of asymmetry of the equation of state for the liquids disappears.

The transformation proposed is different from that used in the Catastrophe Theory for the functions [72,74]. The latter is defined only in the neighbourhood of the critical point. In fact, any $(n-2)$ parametric set of probability densities performing the catastrophe of type $A_{2 n}$ by changing of the variable can be transformed into the canonical form of exponent on the polynomial of the $2 n$ degree. In particular, the probability density $h(x)$ with the only critical point can be transformed into gaussian distribution $g(\tilde{x})=\frac{1}{2 \pi} \exp \left(-\tilde{x}^{2}\right)$ by transformation of the variable $x \rightarrow \tilde{x}$. The details of the realization of this procedure for Ising-like systems are given in [73].

To illustrate the importance of the technique formulated above we give the results for the parameters of the Ising model. The exact forms of the coefficients of the initial effective Hamiltonian for this model equal those for Curie-Weiss approximation (see [75]):

$$
a_{2 n}=\frac{1}{2 n-1}-2 D \beta J \delta_{2, n}
$$

The procedure of the canonical transformation leads to the following results:

$$
\begin{aligned}
& A_{2}(T)=\alpha \tau+o(\tau), \quad A_{4}=0.53, \\
& \text { where } \alpha \approx 1, \quad \tau=\frac{T-T_{c}}{T_{c}} .
\end{aligned}
$$

We see that the coefficient $A_{4}$ differs essentially from $a_{4}=\frac{1}{3}$ while $\alpha$ practically does not change.

It is important that the canonical order parameter $\phi$ is the analytical function of initial order parameter $\eta$ :

$$
\eta=\phi+\frac{1}{2} \Gamma_{2} \phi^{2}+\frac{1}{3} \Gamma_{3} \phi^{3}+\ldots
$$

where the coefficients $\Gamma_{i}$ can be expressed through the coefficients $a_{n}$ of the initial Hamiltonian. Indeed, in the vicinity of the point $\eta=0, \phi=0$ from (114)

$$
\eta+\int_{0}^{\eta} h_{\mathrm{loc}}(x)+\frac{1}{2} \int_{0}^{\eta} h_{l o c}(x)^{2} d x+\ldots=\phi+\int_{0}^{\phi} h_{\mathrm{loc}}^{(\mathrm{can})}(x) d x+\frac{1}{2} \int_{0}^{\phi} h_{\mathrm{loc}}^{(\mathrm{can})}(x)^{2} d x+\ldots
$$

Using

$$
h_{\mathrm{loc}}(\eta)=a_{1} \eta+\frac{1}{2} a_{2} \eta^{2}+\ldots
$$

and (113) we get:

$$
\eta+\frac{1}{2} a_{1} \eta^{2}+\frac{1}{6}\left(a_{2}+a_{1}^{2}\right) \eta^{3}+\ldots=\phi+\frac{1}{2} A_{1} \phi^{2}+\frac{1}{6}\left(A_{2}+A_{1}^{2}\right) \phi^{3}+\ldots
$$

and finally

$$
\Gamma_{2}=A_{1}-a_{1}, \quad \Gamma_{3}=\frac{1}{2}\left(A_{2}+A_{1}^{2}-a_{2}-a_{1}^{2}-3 a_{1} \Gamma_{2}\right), \quad \text { etc. }
$$

Further we assume that the procedure of the reduction of the effective Hamiltonian (105) to the canonical form has been carried out.

\section{INFLUENCE OF THE POLARIZATIONAL EFFECTS ON THE CRITICAL BEHAVIOUR}

\section{A. Ginzburg number for the ionic liquids}

The expressions for $a_{2}^{(\mathrm{pol})}$ and $a_{4}^{(\mathrm{pol})}$, which follow from (83) and (90) read as:

$$
\begin{aligned}
& a_{2}^{(\mathrm{pol})}=9 \frac{\lambda^{2}}{(1+2 \lambda)^{2}(1-\lambda)^{2}} \beta_{c} f_{\mathrm{ch}}^{(\mathrm{eq})} \\
& a_{4}^{(\mathrm{pol})}=\frac{27}{2} \frac{\lambda^{4}}{(1+2 \lambda)^{4}(1-\lambda)^{4}} \beta_{c} f_{\mathrm{ch}}^{(\mathrm{eq})} .
\end{aligned}
$$


It follows from (77) that polarizational contribution to $a_{4}$ is negative, therefore, the Ginzburg number decreases with account of polarization effects. Here the approximation $\Delta \approx \Delta_{c}$ was used. Using the dimensionless form of coefficients of the LGH obtained in $[69,70]$ we calculate the Ginzburg temperature for ionic fluid:

$$
G i=\frac{9 a_{4}^{2}}{8 \pi^{2} \tilde{a}_{2}}\left(\frac{a}{b}\right)^{6},
$$

where

$$
\tilde{a}_{2}=\lim _{\tau \rightarrow 0} \frac{a_{2}}{\tau}, \quad \tau=\frac{T-T_{c}}{T_{c}} .
$$

It is useful to rewrite (127) in the following form

$$
G i=G i_{0}\left(1+\frac{a_{4}^{(\mathrm{pol})}}{a_{4}^{(0)}}\right)^{2}
$$

where $G i_{0}$ is the Ginzburg temperature without accounting of the inhomogeneous polarization. Here we neglect the renormalization of the coefficients $\tilde{a}_{2}$ and $b$ of the LGH. It appears that in this approximation $G i$ slightly decreases in comparison with the initial value because the value of $\lambda$ is actually small $(<0.1)$ since $\epsilon$ close to 1 .

Below the critical point the system separates into liquid and gaseous phases with different densities because of strong density fluctuations. These phases have different degrees of dissociation $\Delta_{\text {liq }}$ and $\Delta_{\text {gas }}$ since thermodynamically the degree of dissociation $\Delta$ is a function of $T^{*}$ and $\rho^{*}$. At the very critical point $\Delta^{(\text {liq })}=\Delta^{\text {(gas) }}$ but below $\Delta_{\text {liq }} \neq \Delta_{\text {gas }}$ because of $\rho_{\text {liq }}^{*} \neq \rho_{\mathrm{g}}^{*}$. From the physical point of view one can expect the new type (nonIsing like asymptotics) for the critical behaviour only if density fluctuations strongly interact with the ones for charge. This interaction occurs only if the dipoles exist since fluctuations of their number are directly connected with fluctuations of the density. Thus the very fact that below critical point $\Delta^{(\text {liq) }} \neq \Delta^{\text {(gas) }}$ means that the fluctuations of the number of neutral pairs are strong and therefore the polarizational effects should be taken into account. Since the density fluctuations are strong the fluctuations of dipole number are strong too. That means that the fluctuations of charge numbers are also strong though mutually correlated due to neutrality condition $\left(\delta n_{+}=-\delta n_{-}\right)$. Indeed, one could expect the peculiarities for the critical behaviour in IL if the degree of dissociation strongly depends on density near the critical point. Therefore, to consider the case of strong interaction between charge and density fluctuations we should not neglect density dependence of $\Delta$ near the critical point. It is clear that this dependence is very essential for the dielectric permittivity as has been noted above. Here we use the linear approximation for such dependence:

$$
\Delta(\rho, \tau)=\Delta_{c}+\Delta_{1} \eta+o(\eta) .
$$

The estimate for $\Delta_{1}$ can be obtained from [12], where we can find that for different $\operatorname{EOS} 0<\Delta_{1}<10$. The coefficients $a_{2}^{(\mathrm{pol})}$ and $a_{4}^{(\mathrm{pol})}$ take the values:

$$
\begin{aligned}
& a_{2}^{(\mathrm{pol})}=9 \frac{\left(\lambda-\Delta_{1}\right)^{2}}{(1+2 \lambda)^{2}(1-\lambda)^{2}} \beta_{c} f_{\mathrm{ch}}^{(\mathrm{eq})} \\
& a_{4}^{(\mathrm{pol})}=\frac{27}{2} \frac{\left(\lambda-\Delta_{1}\right)^{4}}{(1+2 \lambda)^{4}(1-\lambda)^{4}} \beta_{c} f_{\mathrm{ch}}^{(\mathrm{eq})} .
\end{aligned}
$$

Note that (131) corresponds to the linear approximation for the $\epsilon(\eta)$ dependence. The main result, the diminishing of the Ginzburg temperature, appears in this approximation. Actually to construct $a_{4}^{\text {(pol) }}$ the terms up to the 4 -th order in $\eta$-expansion for $\epsilon(\eta)$ should be included. Sure the explicit expressions for $a_{2}^{(\mathrm{pol})}$ and $a_{4}^{(\mathrm{pol})}$ become very complex and we will not give them here. These results are represented in Fig. 3.

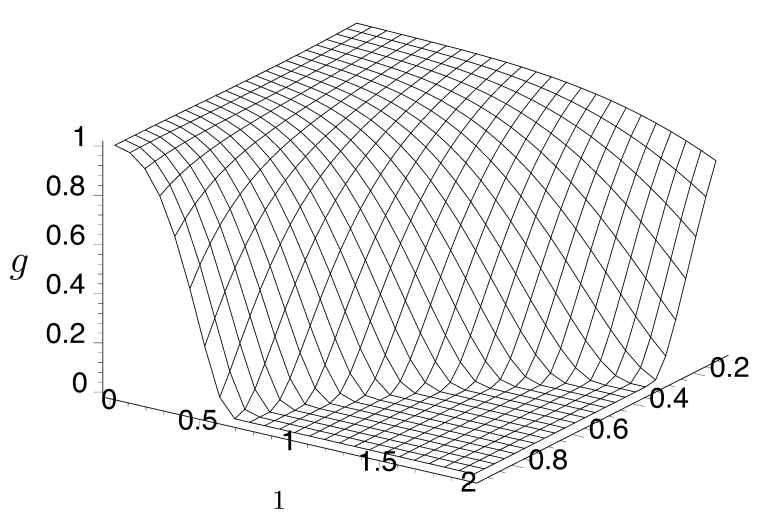

Fig. 3. The dependence of the Ginzburg temperature $g=G i / G i_{0}$ obtained with (90) on the degree of ionization $\Delta$ and $\Delta_{1}$ at $a_{4}^{(0)}=0.01, \delta=1, \rho_{c}^{*} / T_{c}^{*}=0.5$.

The key feature is the vanishing of the Ginzburg number at low dielectric permittivity values and small screening length while the value of $\Delta_{1}$ is high enough and $\Delta$ is close to 1 . This region of parameters naturally corresponds to the state of the ionic liquid with high degree of dissociation at the critical point. Thus, the account of polarizational effects caused by the density fluctuations is very essential for analysis of asymptotical behaviour of ionic fluids which was pointed out in [41]. Using other model equations of state like nonlimiting DH (nDH) approximation (14) and MSA equation (21) for constructing the LGH does not change the results significantly. Namely, the difference between the results obtained with the help of equations (79) and (21) does not exceeds $5 \%$. DH approximation gives qualitatively the same results with the difference in comparison with MSA and $\mathrm{nDH}$ EOS up to $20 \%$ for $\Gamma_{c}<0.3$. It directly follows from (76) which shows that all polarizational corrections to the LGH is proportional to $\beta \delta f_{\text {ch }}^{(e q)}$. Since DH EOS is 
valid only for low density systems the usage of this EOS should be treated as mere illustrative.

For the comparison we also give the results (see Fig. 4) for the Ginzburg temperature calculated basing on the Onsager formula for the dielectric permittivity [77]:

$$
\epsilon=\frac{1}{4}\left(1+3 x+3 \sqrt{1+\frac{2 x}{3}+x^{2}}\right)
$$

where

$$
x=\frac{2 \pi d^{2}\left(1-\Delta-\Delta_{1} \eta\right)}{3 T^{*}}
$$

As follows from the obtained results for $G i$ the steep increase of the degree of ionization with density may result in a significant lowering of $G i$. For sufficiently great values of $\Delta_{1}=\frac{\partial \Delta}{\partial \eta}$ the Ginzburg temperature may vanish. The value of $\Delta_{1}$ at which $G i=0$ increases if the locus parameter $\frac{\rho_{c}^{*}}{T_{c}^{*}}$ decreases. This is quite natural since for the Coulombic criticality to happen at low density the stronger density dependence for the degree of dissociation is needed.

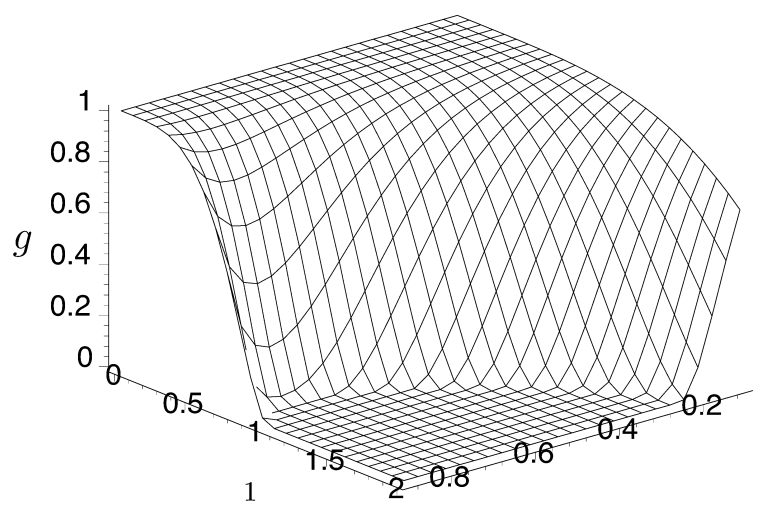

Fig. 4. The dependence of the Ginzburg temperature $g=G i / G i_{0}$ obtained with (132) on the degree of ionization $\Delta$ and $\Delta_{1}$ at $a_{4}^{(0)}=0.01, \delta=1, \rho_{c}^{*} / T_{c}^{*}=0.5$.

In other words the Coulombic driven criticality is characterized by a small value of $G i$ caused by strong density dependence of the degree of dissociation at the critical region. The following scenarios are possible:

1. $\Delta$ is continuous at the critical point but $\left.\frac{\partial \Delta}{\partial \rho}\right|_{\rho^{*}=\rho^{*}, T=T_{c}^{*}}$ is very large. Note that due to very low estimates for $\rho_{c}^{*}$ in different mean field approximations even if $\Delta_{1} \simeq 1$ the value of $\left.\frac{\partial \Delta}{\partial \rho}\right|_{\rho^{*}=\rho_{c}^{*}} \simeq 10^{2}$. Here the anomaly small value of $G i$ is observed but the critical behaviour is Ising-like. The polarizability of a system also renormalizes the coefficient $b$. As has been noted above the Coulombic interactions prevent the spatial separation of op- posite charges in a system. Therefore, in quasilocal approximation the energy of a system with inhomogeneous dielectric permittivity should be higher than that for the homogeneous one. In other words, the polarization contribution increases the value of $b$. Thus, we obtain the upper estimate for $G i$.

2. $\Delta$ is continuous at the critical point but its fluctuations are essential $\sqrt{\left\langle(\delta \Delta)^{2}\right\rangle} \simeq\langle\Delta\rangle$. In general $\Delta$ is the sum thermodynamical equilibrium part $\Delta^{(\mathrm{eq})}\left(\rho^{*}, T^{*}\right)$ and fluctuation one $\Delta^{(\mathrm{fl})}: \Delta=$ $\Delta^{(\mathrm{eq})}\left(\rho^{*}, T^{*}\right)+\Delta^{(\mathrm{fl})}$. Therefore the quantity $\Delta_{\text {liq }}-$ $\Delta_{\mathrm{g}}$ includes the part orthogonal to the density fluctuations and can be considered as concurrent order parameter and a new type of critical behaviour distinct from Ising like can be expected. In particular, if the coefficient at the gradient term $c(\partial \Delta)^{2}$ tends to zero the spatial inhomogeneous phase with respect to $\Delta$ and possibly the density is expected. The analogue of such a phase in Condense Matter is the excitonic drops [60]. In such a case the initial liquid-vapour critical point can transform to peculiar point similar to Lifshitz one [39]. Note that spatial inhomogeneity of $\Delta$ in no way means the spatial separation of charges i.e., charge density wave phase. In addition this scenario is closely connected with metal-insulator transition [33].

3. The discontinuity of density dependence of $\Delta$ at the mean field critical point. According to the definition at the point of the second order phase transition the difference between phases disappear. If there is a discontinuity in $\Delta$ at the critical point then it is not the second order phase transition. This case needs detailed investigation.

Note that a spatially modulated charge density and order parameter waves was predicted in [66]. There was considered a completely different system namely electrolyte solution of small concentration, with strong contact interaction between neutral density fluctuations and charged subsystem. However, similar character of intermode interaction cannot be justified. Unlike of this the possibility of appearance the microhomogeneous state in molten salt $\mathrm{NaCl}$ is connected with realistic polarizational effects and independent (uncorrelated) fluctuations of the degree of dissociation do not lead to spatial separation of charges. The case of repulsive hard-core driven criticality $[5,6]$, is characterized by insignificant change in $G i$ and weak density dependence of degree of dissociation.

\section{B. Ginzburg criterion for electrolyte solutions}

It is difficult to solve the problem of the type of critical behaviour of a system in experiments. The main question here is the estimation of the Ginzburg number, which controls the width of the asymptotic region $t_{\times}$. Its magnitude depends on the microscopic parameters of a system and as follows from experiments varies in a wide range. In [22] the crossover from classical to Ising-like behaviour was clearly observed at $t_{\times} \approx 10^{-2}$. In $[2,19]$ the 
data were fitted by classical asymptotics up to $t_{\times} \approx 10^{-4}$ near the critical point. The molecular dynamics simulations of molten salt [32] also show classical behaviour of an order parameter for $\tau \approx 10^{-2}, \tau=\frac{T-T_{c}}{T_{c}}$, although the influence of finite size effects does not allow to say exactly whether the crossover does take place or not. In all cases the width of fluctuation region is smaller than the one for molecular liquids. In [23] the dependence of the width of crossover region on dielectric permittivity $\epsilon$ of a solvent was investigated. It was observed that the crossover region becomes smaller as $\epsilon$ is decreased. There are not any physical reasons for the existence of such $\epsilon^{*}$ that $G i(\epsilon) \rightarrow 0$ as $\epsilon \rightarrow \epsilon^{*}>1$. In case of $\epsilon^{*}=1$ we deal with the plasma phase transitions [78].

In previous section we have constructed the effective LGH for electrolyte solution near its vapour-liquid critical point. It is well known [79] that the crossover from classical to Ising-like critical behaviour of a system occurs at the temperature:

$$
\tau \approx G i
$$

where

$$
G i=\frac{a_{4}^{(c)^{2}} T_{c}^{2}}{\alpha b^{3}}, \quad \alpha=\left.\frac{d a_{2}^{(c)}}{d \tau}\right|_{T=T_{c}}
$$

Values $\alpha_{0}\left(a_{2}^{(0)}=\alpha_{0} \tau\right)$ and $a_{4}^{(0)}$ for initial Hamiltonian can be extracted from the van der Waals equation (see [71]):

$$
\alpha_{0} \simeq \beta_{c} \Phi \delta^{2}, \quad a_{4}^{(0)} \simeq \delta^{4}
$$

where $\delta=n_{c} \sigma^{3}$ is the dimensionless density and $-\Phi$ is the minimum value of the interparticle potential for a solvent. Using (108) yields:

$$
\begin{aligned}
\frac{a_{4}^{(c)}}{a_{4}^{(0)}} & =1-\tilde{a}_{4} x^{3 / 2}+o\left(x^{3 / 2}\right) \\
\frac{\alpha}{\alpha_{0}} & =1+\tilde{\alpha} x^{3 / 2}+o\left(x^{3 / 2}\right) \\
\frac{b}{c_{0}} & =1+\lambda_{1} x^{\frac{1}{2}}+o\left(x^{\frac{1}{2}}\right)
\end{aligned}
$$

where

$$
\begin{aligned}
\tilde{a}_{4} & \simeq \frac{1}{a_{4}^{(0)}}\left(\Gamma_{*} \sigma\right)^{3} \delta^{4}\left(\frac{\partial \ln \epsilon}{\partial \ln \rho}\right)^{4} \simeq 0.1 \div 1 \\
\tilde{T} & =\frac{T_{c}-T_{c}^{(0)}}{T_{c}^{(0)}} \simeq 0.01 \\
\tilde{\alpha} & \simeq \frac{1}{\alpha_{0}}\left(\Gamma_{*} \sigma\right)^{3} \delta^{2}\left(\frac{\partial \ln \epsilon}{\partial \ln \rho}\right)^{2} \simeq 1
\end{aligned}
$$

$$
\lambda_{1} \simeq \frac{1}{c_{0}}\left(\sigma \Gamma_{*}\right) \simeq 0.1
$$

The numerical estimations for $\tilde{a}_{4}, \ldots \lambda_{1}$ are obtained for the following values of parameters:

$$
\delta \simeq 0.33, \quad \beta_{c} \Phi \simeq 1
$$

We assume that $\frac{\partial \ln \epsilon}{\partial \ln p}$ can be approximated by the formula:

$$
\frac{\partial \ln \epsilon}{\partial \ln \rho}=\frac{A \rho}{\epsilon}
$$

and the coefficient $A$ is equal to $280 \frac{\mathrm{cm}^{3}}{\mathrm{~g}}$ for water in accordance with [81]. At least by the order of magnitude $\Gamma_{*} \sigma<1$. Using (134) and (136) we obtain the following renormalized value of the Ginzburg number:

$$
G i(x) \approx G i^{(0)} \frac{\left(1-\tilde{a}_{4} x^{3 / 2}\right)^{2}}{\left(1+\tilde{\alpha} x^{3 / 2}\right)\left(1+\lambda_{1} x^{\frac{1}{2}}\right)^{3}}
$$

where $G i^{(0)}$ is the Ginzburg number for a solvent. In fact the Ginzburg number essentially depends on the ratio of the amplitude of correlation length $r_{0}$ and the Debye screening length. In accordance with said above the Ginzburg number is a monotone decreasing function of concentration. Besides this it also depends on the temperature as a parameter. Summarizing our arguments for the regions $r_{\mathrm{D}}<(>) r_{c}$ we can write:

$$
G i(x \mid \tau)=\left\{\begin{array}{ll}
G i^{(0)}, & x \ll x_{\mathrm{D}}(\tau) \\
G i(x), & x_{\mathrm{D}}(\tau) \ll(<) x
\end{array},\right.
$$

where

$$
x_{\mathrm{D}}(\tau)=\left(\frac{1}{\Gamma_{*} r_{0}}\right)^{2} \tau^{2 \nu}
$$

is the limit concentration of an electrolyte, which determines the applicability region of our polarization model. The concentration dependencies of $G i$ at some fixed $\tau$ and different values of dielectric permittivity are presented on Fig. (5).

Note that the greater is the value of dielectric permittivity the greater is $G i$. This fact was noted experimentally in [23]. Fig. (6) shows the concentration dependence of $G i$ for electrolyte solution with $\epsilon=80$ and $\Gamma_{*} \sigma=0.5$. The qualitative behaviour of $G i(x)$ near $x_{\mathrm{D}}$ is shown by dashed line.

Essentially that the value of $a_{4}$ vanishes and system looses its stability at concentration of an electrolyte:

$$
x_{*}=\tilde{a}_{4}^{-\frac{2}{3}}
$$


Obviously this possibility realizes only if $\tilde{a}_{4}>1$ (see (136)). In this case one can expect the multicritical behaviour of a system [6].

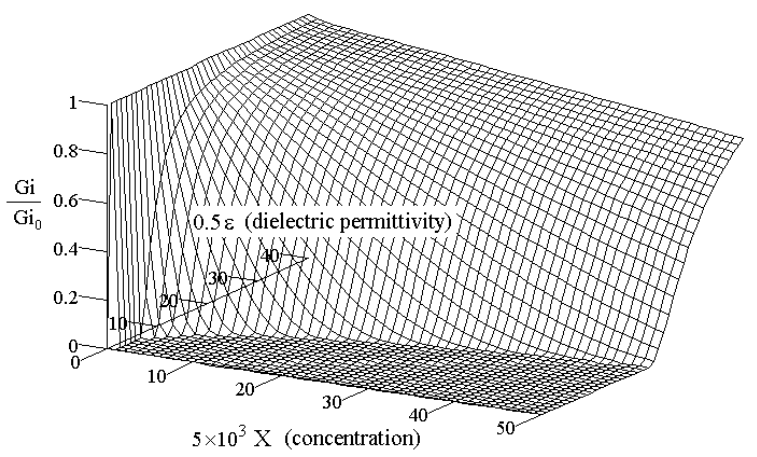

Fig. 5. The relative value $\frac{G i}{G i_{0}}$ of the Ginzburg number as a function of concentration ( $x$-axis) and dielectric permittivity (y-axis) calculated by formula (139). The scales on $x$ and $y$ axes equal to $1: 0.0002$ and $1: 2$ respectively.

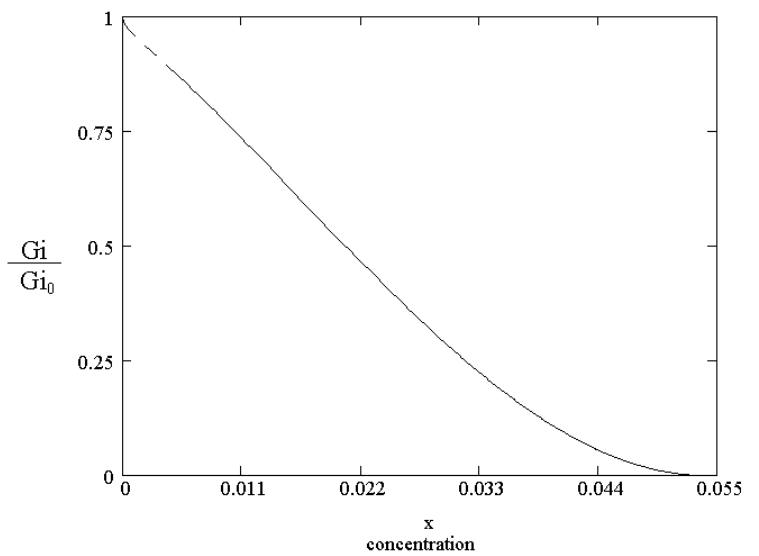

Fig. 6. Concentration dependence of the Ginzburg number for parameters given in text.

Using the formula (140) is not convenient since usually experiments are carried out at a fixed concentration. Let us illustrate this situation considering the values of critical exponent. According to (140) we have:

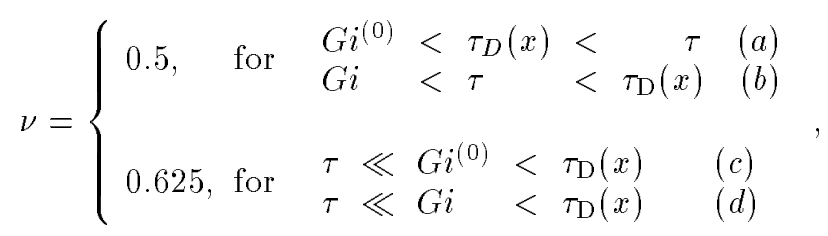

$$
\tau_{D}=\left(r_{0} \Gamma_{*} \sqrt{x}\right)^{1 / \nu} \sim x
$$

At the end of this section let us complete obtained results with qualitative arguments. By order of magnitude [79]

$$
G i=\left(\frac{r_{s}}{r_{0}}\right)^{6}
$$

where $r_{s}$ is the interparticle spacing. Addition of an electrolyte leads to augmentation of the correlation length amplitude because of its renormalization by chargecharge fluctuations. It is clear that the behaviour of strongly concentrated electrolytes is expected to be similar to that of simple liquids or liquid metals.

\section{Remarks}

The important role of polarizational effects in the critical behaviour of ionic melts has been demonstrated. It is established that if the key parameters of a system take the values: $r_{s}=1 \div 5, \Delta_{1}>0.5$, the coefficient $a_{4}$ of the effective LGH reduces considerably or vanishes.

Similar situation is also characteristic for the critical behaviour of electrolyte solutions. In them the charge fluctuations of admixtured ions can essentially renormalize the coefficient $a_{4}^{(0)}$ of the initial LGH for a solvent. At definite concentration $x_{*}$ of electrolyte $a_{4}\left(x_{*}\right)=0$. For higher concentrations the standard scenario of the critical behaviour becomes inapplicable and additional investigations are necessary. In connection with this we note the result obtained experimentally in [15], for ternary aqueous solution of sodium bromide. It was observed that fluctuation region at salt concentration 0.17 (mass fraction) less than $10^{-5}$. To interpret this as well as specific dip on the line of lower critical points the conjecture about existence of competing microheterogeneous phase was put forward. In principle, it is possible that at some concentration the line of the lower critical points may contact the virtual phase spinodal whose branches are directed to lower temperatures. Then between the branches of the spinodal the state of electrolyte solution should be heterogeneous according to the thermodynamic demands.

The main peculiarities of the critical behaviour of molten $\mathrm{NaCl}$ salt are determined by the density dependence of the degree of dissociation $\Delta$ and its fluctuations. When the fluctuations of $\Delta$ are relatively small, the critical behaviour of the ionic liquid should be Ising-like. From the thermodynamic point of view it is supported by the fact that the system is characterized by two thermodynamic degrees of freedom [72]. The specificity of the system displays only in the numerical value of the Ginzburg number: it is the less the more the derivative $\left.\frac{\partial \Delta}{\partial \rho}\right|_{T}$ is.

If the fluctuations of $\Delta$ become strong, $\sqrt{\left\langle(\delta \Delta)^{2}\right\rangle} \simeq$ $\langle\Delta\rangle$, the deviations from the Ising-like behaviour can be 
considerable. First of all, the system can demix on the parameter $\Delta$. In other words, the formation of drops with different values of $\Delta$ is possible. Since $\Delta$ is crucial parameter, the change of the type of the critical behaviour seems to be probable.

It is not excluded that the phase diagram of molten salt $\mathrm{NaCl}$ near its liquid-vapour critical point will be more complex in comparison with that for onecomponent liquid with simple intermolecular interaction. In particular, the derivative $\left.\frac{\partial^{3} P}{\partial n^{3}}\right|_{T=T_{C}}$ can be close to zero, that can lead to the change of the type of the critical point, e.g., tricritical behaviour. However the vanishing of $a_{6}$ is impossible. The behaviour of conductivity can serve as additional test of the type of the critical behaviour. So the sharp change of the conductivity could testify the point of phase transition different from the second order.

The most crucial for the critical behaviour is the dependence of $a_{4}$ on the degree of dimerization $\Delta$ of the system which directly influences the polarizability. The density of the ionic liquid is formed by the density of free charges and bounded states. The density of the nondissociated molecules (dipoles) is determined by the thermodynamical parameters of the state of the system (e.g., temperature and specific volume). In particular, the results of Monte Carlo simulations indicate that the proximity of these two transition could explain the crossover phenomena in ionic fluids [33]. Note that our consideration is based on mean field treatment. Therefore, the thorough analysis of fluctuation effects is needed to determine the type of the critical behaviour if $a_{4}=0$.

\section{SPECIFIC EFFECTS}

\section{A. Fluctuation-induced shift of the critical point in solution of electrolytes}

An anomalous curvature of the $T-x$ and $P-x$ projections of the critical line of electrolyte solution at very small mole fractions of an electrolyte $\mathrm{NaCl}+\mathrm{H}_{2} \mathrm{O}$ was observed in many experimental works $[26,82,83]$. But the nature of very big values of $\frac{d T_{c}(x)}{d x}$ was not clarified.

We will show that the shift of the critical point of high diluted solution from the locus of pure solvent is governed predominantly by the charge-charge fluctuations. The 'square root' concentration law for such a shift is a direct consequence of the polarizational charge-density coupling theory proposed in previous sections. The dependence of the coefficients of the effective LGH at low concentrations is given by Eq. (136). In mean field approximation the shift of the critical temperature as it follows from Eq. (136) is

$$
\Delta^{(\text {mean })} T_{c} \simeq x^{3 / 2}
$$

But the fluctuations change slightly the value of the critical temperature. This fluctuation-induced shift of the critical temperature (the locus of the critical point in general) is proportional to

$$
\Delta^{(\mathrm{fl})} T_{c} \simeq \sqrt{x}
$$

Indeed, let us define renormalized order parameter so that the coefficient at the gradient term equals to unity:

$$
\phi(\mathbf{r})=\sqrt{c(x)} \eta(\mathbf{r})
$$

The LGH for the new order parameter reads as follows

$$
\beta H_{\mathrm{eff}}[\phi]=\int d V\left(\frac{1}{2}(\nabla \phi(\mathbf{r}))^{2}+\tilde{a}_{1}(T, x) \phi(\mathbf{r})+\frac{1}{2} \tilde{a}_{2}(T, x) \phi^{2}(\mathbf{r})+\frac{1}{4} \tilde{a}_{4}(T, x) \phi^{4}(\mathbf{r})\right)
$$

where

$$
\tilde{a}_{k}(T, x)=(c(x))^{-k / 2} a_{k}(T, x)
$$

In the first order of perturbation theory on fluctuation coupling constant $\tilde{a}_{4}$ [84] we have

$$
\tilde{a}_{2}^{R}(T, x)=\tilde{a}_{2}(T, x)+\left.3 \tilde{a}_{4}(T, x) \frac{d}{d \Lambda} \int_{0}^{\Lambda} G_{0}(\mathbf{q}) d \mathbf{q}\right|_{\Lambda=1}, \quad G_{0}(\mathbf{q})=\frac{1}{\tilde{a}_{2}(T, x)+\mathbf{q}^{2}}
$$


From Eq. (147) it follows that

$$
\frac{d T_{c}(x)}{d x} \sim x^{-1 / 2}
$$

which explains the anomalously big values of $\frac{d T_{c}(x)}{d x}$ observed in $[26,82,83]$. Note, that such a term was used pure empirically in [85] to process the experimental data. The same conclusion on existence of $\sqrt{x}$ terms is valid for other coordinates of the critical point such as pressure and density.

\section{B. Asymmetry of a binodal for electrolyte solutions}

In this section we will show that the addition of electrolyte leads to essential additional asymmetry of the vapour-liquid coexistence curve. This circumstance is connected with the choice of appropriate order parameter, which restores the symmetrical shape of a binodal. Otherwise, the $\sim \tau^{2 \beta}$ term, which is absent in standard variants of the asymptotic equation of state [79] should be introduced to fit the experimental data in laboratory variables [80]. We establish this fact in an evident form within the framework of the canonical formalism $[71,86]$, which gives a clear motivation for all standard asymptotic terms of the equation of state as well as for those of form $\tau^{n \beta}$, introduced to process the experimental data.

As it follows from the scale-invariant theory of critical phenomena the singular part of the equation of state is given by the expression

$$
\langle\eta\rangle=\left|h_{2}\right|^{\beta} g_{s}\left(\frac{h_{1}}{\left|h_{2}\right|^{\beta+\gamma}}\right) \text {. }
$$

The order parameter $P$ and conjugated fields $h_{1}, h_{2}$ within the canonical formalism should be identified with $\phi$ and the coefficients $A_{1}, A_{2}, A_{4}$ of the Hamiltonian (113). As a result, the equation (153) takes the form

$$
\langle\phi\rangle=\left|A_{2}\right|^{\beta} g_{s}\left(\frac{A_{1}}{\left|A_{2}\right|^{\beta+\gamma}}\right) .
$$

Here the brackets $\langle\ldots\rangle$ designate the averaging on the volume of the correlation sphere $\left(\propto r_{c}^{3}\right)$. In particular, the equation of binodal including the additional Wegner's term [87] is as follows

$$
\langle\phi\rangle_{\text {bin }}= \pm\left|A_{2}^{*}\right|^{\beta} g_{s}(0)\left(1+b_{2}\left|A_{2}^{*}\right|^{\Delta}+\ldots\right)
$$

where $A_{2}^{*}=\left.A_{2}\right|_{A_{1}=0}$. Its 'liquid' and 'gas' branches in variables $\left(\psi, a_{2}^{*}\right)$, as is clear from Eq. (154) are absolutely symmetric. But this symmetry disappears if we return to the initial ('laboratory') variables $(\eta, \tau)[71,86]$. Indeed, using (121) one can get:

$$
\langle\eta\rangle=\langle\phi\rangle+\frac{1}{2} \gamma_{2}\left\langle\phi^{2}\right\rangle+\ldots
$$

Since (see [79])

$$
\left\langle\phi^{2}\right\rangle=\langle\phi\rangle^{2}+\left|h_{2}\right|^{1-\alpha} l_{s}\left(\frac{h_{1}}{\left|h_{2}\right|^{\beta+\gamma}}\right) \text {, }
$$

where function $l_{s}(x)$ is inverse to $g_{s}(x)$, Eq. (156) in asymptotic region transforms to:

$$
\begin{aligned}
\frac{1}{2}\left(\langle\eta\rangle_{\text {bin }}^{+}-\langle\eta\rangle_{\text {bin }}^{-}\right) & =g_{s}(0)\left|a_{2}^{*}\right|^{\beta}(1 \\
& \left.+b_{2}\left|a_{2}^{*}\right|^{\Delta}+\ldots\right)+\ldots \\
\frac{1}{2}\left(\langle\eta\rangle_{\text {bin }}^{+}+\langle\eta\rangle_{\text {bin }}^{-}\right) & =\frac{1}{2} \Gamma_{2}\left[g_{s}^{2}(0)\left|a_{2}^{*}\right|^{2 \beta}\right. \\
& \left.+l_{s}(0)\left|a_{2}^{*}\right|^{1-\alpha}\right]^{1-\ldots}+\ldots
\end{aligned}
$$

Note that the equation (157) besides standard terms [79] includes additional contribution $\propto|\tau|^{2 \beta}$ (as well as other terms $\propto|\tau|^{n \beta}, n>2$ ). The latter was introduced in work $[80]$ from the empirical reasons. This new term with $\beta=0.5$ appears in mean-field approximation [39] as well. Essentially, the asymmetry of the coexistence curve is determined by product of universal multipliers $g_{s}(0)$ and $l_{s}(0)$ and coefficient $\Gamma_{2}$ describing the individual properties of liquids and solutions. The temperature and concentration dependencies of coefficients $\Gamma_{2}, a_{2}^{*}$ are determined by the expressions (108), (125) and can be represented as follows:

$$
\begin{aligned}
& \Gamma_{2} \simeq \Gamma_{2}^{(0)}+\Gamma_{2}^{(\mathrm{el})}, \\
& a_{2}^{*} \simeq a_{2}^{(0)}+a_{2}^{(\mathrm{el})},
\end{aligned}
$$

$\Gamma_{2}^{(0)}, a_{2}^{(0)}$ are the values of the respective coefficients in absence of electrolyte. The values of terms induced by electrolyte impurity in Eq. (158) are of $\propto x^{3 / 2}$ order and strongly depend on the parameter $\Gamma_{*} \sigma$. If $\Gamma_{*} \sigma<(\ll) 0.1$ they can be omitted. In the opposite cases, an account of additional terms is rather essential.

\section{Conductivity of the electrolytes}

Above it was shown that the peculiarities of the critical behaviour of IL are determined by the density dependence $\Delta\left(\rho^{*}\right)$ of the degree of dissociation near the critical point. The criticality of the conductivity $\sigma$ for highly concentrated ionic mixtures and other electrolytes has been studied much less compared with their equilibrium thermodynamical properties [1,88]. The measurements of the conductivity for highly concentrated nonaqueous electrolytes was presented in [88].

It is well known (see [79]) that for magnetic systems with Ising symmetry for an order parameter and conjugated field the critical fluctuations lead to a singularity for the conductivity $\sigma$ similar to that of entropy on the critical isochor [79]: 


$$
\sigma=\sigma_{c}+A_{r} \tau+A_{s} \tau^{1-\alpha}+\ldots
$$

Formally the appearance of the singular term is easily explained with the help of a thermodynamic relation between the variations of the conductivity, $\delta \sigma$, and the entropy, $\delta s$,

$$
\delta \sigma \propto-\omega_{\mathrm{rel}}(\mathbf{k}=0) \frac{T}{\left\langle\mathbf{j}^{2}\right\rangle} \sigma^{2} \delta s,
$$

where $\omega_{\text {rel }}$ is the characteristic relaxation rate for the conductivity, provided that it is nonzero at the critical point, i.e., no critical slowing down for the conductivity occurs. This is certainly true for scenario 1 above. Let us consider this case in more detail focusing on the connection of the singular term for the conductivity with the behaviour of the key parameter $\Delta$. As is known, the conductivity of a system is determined by

$$
\sigma=\frac{\beta}{3} \int_{0}^{\infty} \int_{V}\langle\mathbf{j}(\mathbf{r}, t) \cdot \mathbf{j}(0,0)\rangle d \mathbf{r} d t
$$

where $\mathbf{j}(\mathbf{r}, t)$ is the electrical current density,

$$
\mathbf{j}(\mathbf{r}, t)=e \rho_{\mathrm{ch}}(\mathbf{r}, t) \mathbf{v}(\mathbf{r}, t)
$$

and $\rho_{\mathrm{ch}}(\mathbf{r}, t)$ is the density fluctuation of charged component. The overall density $n$ is a sum of the density of charged component (free carriers) and the double density of dipole pairs. The density fluctuation is the sum of the fluctuations of these terms. To calculate (161) we can use the arguments of [89]. Due to screening effect, the correlation of charge fluctuations separated by distance $r>r_{s}$ is negligible. Due to this, in DH approximation we get

$$
\left\langle\rho_{\mathrm{ch}}(\mathbf{r}, t) \rho_{\mathrm{ch}}(0,0)\right\rangle \approx \frac{1}{4 \pi \beta r_{s}^{2}}\left(\frac{\partial \rho_{\mathrm{ch}}}{\partial \mu}\right)_{T} \frac{e^{-r / r_{s}}}{r} f\left(\omega_{\mathrm{osc}}\right),
$$

where

$$
\frac{\partial \rho_{\mathrm{ch}}}{\partial \mu}=n_{c} \frac{\partial \Delta}{\partial \mu}+\Delta_{c} \frac{\partial n}{\partial \mu} .
$$

$n$ is the overall density and $f$ is a function without singularities. We will not be interested in time relaxation of charge fluctuations here. The peculiarities of the critical behaviour of the conductivity are mainly determined by the derivative $\frac{\partial \rho_{c h}}{\partial \mu}$. Since the number of charges is $N=N_{0} \Delta(\rho, T)$ the singularity of $\left.\frac{\partial \Delta}{\partial \mu}\right|_{T=T_{C}}$ can be obtained from the analysis of $\left.\frac{\partial N}{\partial \mu}\right|_{T_{c}}$. The last is given by the thermodynamic identity (see, e.g., [39]):

$$
\begin{aligned}
& \left(\frac{\partial N}{\partial \mu}\right)_{T}=\frac{\left(\frac{\partial N}{\partial T}\right)_{\mu}^{2}}{\left(\frac{\partial S}{\partial T}\right)_{\mu}-\frac{C_{V}}{T}}, \quad C_{V}=T\left(\frac{\partial S}{\partial T}\right)_{V, N}, \\
& T=T_{c}(1+\tau)
\end{aligned}
$$

where in the vicinity of the critical point $C_{V}=C_{V}^{(\text {reg) }}+$ $C_{V}^{(\text {sing) }}$, where on the critical isochor $C_{V}^{(\text {sing) }} \propto \tau^{-\alpha}$. The value $\left.\frac{\partial N}{\partial \mu}\right|_{T_{c}}$ is nonzero because of the condition of ionization equilibrium. Therefore, the leading divergent terms in the denominator cancel out, but other less singular terms such as $\tau^{1-\alpha}$ do not. The latter terms are responsible for the singular terms in the conductivity.

\section{CONCLUSION}

In our review we touch upon two questions, characteristic for critical phenomena in IL and electrolyte solutions: 1) the basic model for IL and 2) the crossover problem for the critical exponents in electrolyte solutions and IL. We have paid the attention for the dipole fluid as the alternative model for the equation of state for the IL. It was shown that the rotation of dipole molecules is very important fact, which should be taken into account. It was established that the definitive influence on the value and behaviour of the Ginzburg number is caused by socalled polarizational interactions. The nature of the lasts for electrolyte solutions and molten salts was discussed in detail. The possibility for the formation of spatially inhomogeneous states near the critical point is considered.

At the same time many important problems were not included in our analysis. First of all, the associationdissociation processes need more careful investigation both near the critical point and far away from it. Probably including the quantum effects can influence the estimates of relevant parameters. The polarizational interactions are also very important for the description of asymmetry effects in the equation of state. The consecutive using of the canonical formalism [73] also is very important for this purpose. This formalism is also important for ionic micellar solutions [90,80]. The polarizational effects should play very important role for the quasibynary solutions for which the addition of electrolyte impurities leads to the appearance of double critical points and phase separation [91]. In those cases, when the coefficient $a_{4}$ is small, the peculiarities of critical fluctuations should be described with the help of RG-method applied to $\phi^{6}$ model. The detailed investigation of the dielectric permittivity is also very important for the accurate solution of problems of the critical behaviour.

\section{ACKNOWLEDGMENTS}

The authors thank Prof. W. Schröer for fruitful discussion of obtained results. We are also grateful to Prof. Yu. Holovatch for the discussion and proposition 
to write this review. The authors appreciate R. Stepanyan and R. Bezdenezhnykh for their help in creating the figures.

\section{APPENDIX: CANONICAL FORM OF THE DIELECTRIC PERMITTIVITY}

There exist many approaches to the problem of dielectric permittivity. Based on different assumptions they allow to describe with the most completeness only one or several characteristic contributions. Unfortunately, too hasty usage of model conceptions lead to errors which are hard to control. In particular, this can change the character of inequalities and essentially influences the values of the density derivatives of dielectric permittivity. Therefore, the discussion of general structure of dielectric permittivity as well as the nature of main contributions to it seems to be appropriate. By definition, dielectric permittivity for an isotropic medium [77] is equal to

$$
\epsilon-1=4 \pi \frac{P}{E}
$$

where $P=|\mathbf{P}|, \mathbf{P}$ is the polarizability vector, and $\mathbf{E}$ is the strength of the Maxwell electric field $(\mathbf{P} \| \mathbf{E})$. In general, we should calculate $\mathbf{P}$ and $\mathbf{E}$ as functions of the external field strength $\mathbf{E}_{0}$. However, for a specimen of the spherical shape the connection between $\mathbf{E}$ and $\mathbf{E}_{0}$ is especially simple:

$$
\mathbf{E}=\frac{3}{\epsilon+2} \mathbf{E}_{0}
$$

Therefore, we can write

$$
\frac{\epsilon-1}{\epsilon+2}=\frac{4 \pi}{3} \frac{P\left(E_{0}\right)}{E_{0}} \equiv \frac{4 \pi}{3} n \alpha_{e f f}
$$

Since the effective polarizability $\alpha_{\text {eff }}$ is a characteristic of a medium, but not of the shape of the specimen Eq. (A3), connecting $\epsilon$ and $\alpha_{\text {eff }}$, is of general character. The left side of (A3) is always less than unity, so the inequality

$$
\frac{4 \pi}{3} n \alpha_{\mathrm{eff}}<1
$$

holds good.

To make a further conclusion about $\alpha_{\text {eff }}$ and $\epsilon$, we rewrite the formula (A3) in the form

$$
\frac{\epsilon-1}{\epsilon+2}=\frac{4 \pi}{3} \frac{\langle\mathbf{D}\rangle_{0}+\frac{1}{3}\left\langle\mathbf{D}^{2}\right\rangle_{0} E_{0}}{V E_{0}}
$$

where $\mathbf{D}$ is the dipole moment of a system, $V$ is its volume, and the angular brackets designate the average over the equilibrium Gibbs distribution. Note that the matter within the spherical example is homogeneously polarized.
In general, the dipole moment has the structure:

$$
\mathbf{D}=\mathbf{D}_{0}+\hat{\alpha} \mathbf{E}_{0}
$$

where $\mathbf{D}_{0}$ is the dipole moment of the isolated system and $\hat{\alpha}$ is its polarizability. Following I. Fisher (unpublished lecture, Odessa University, 1978) $\hat{\alpha}$ can be represented in a form

$\hat{\alpha}=\sum_{i=1}^{N} \sum_{k=1,2} \hat{\alpha}_{1}^{(k)}\left(\mathbf{r}_{i}\right)+\sum_{1 \leq i, j \leq N} \sum_{k_{1}, k_{2}=1,2} \hat{\alpha}_{2}^{k_{1}, k_{2}}\left(\mathbf{r}_{i}, \mathbf{r}_{j}\right)+\ldots$

where $\hat{\alpha}_{1}^{(k)}\left(\mathbf{r}_{i}\right)$ is the tensor of one-particle polarizability for the $i$-th ion of type $k, \hat{\alpha}_{2}^{\left(k_{1}, k_{2}\right)}\left(\mathbf{r}_{i}, \mathbf{r}_{j}\right)$ is the tensor of irreducible two-particle polarizability for $i$-th and $j$-th ions of types $k_{1}$ and $k_{2}$, correspondingly, and so on. A similar expansion is characteristic for the dipole moment:

$$
\mathbf{D}_{0}=\sum_{1 \leq i, j \leq N} \sum_{k_{1}, k_{2}=1,2} \mathbf{d}_{2}^{\left(k_{1}, k_{2}\right)}\left(\mathbf{r}_{i}, \mathbf{r}_{j}\right)+\ldots
$$

Note that within such an approach the central problem is the calculation of irreducible contributions of different orders to $\hat{\alpha}$ and $\mathbf{D}$ but not the problem of the acting field [77]. From symmetry reasons it follows that:

$$
\left\langle\hat{\alpha}_{1}^{(k)}\left(\mathbf{r}_{i}\right)\right\rangle_{0}=\alpha_{1}^{(k)} \hat{I}
$$

$$
\left\langle\hat{\alpha}_{2}^{\left(k_{1}, k_{2}\right)}\left(\mathbf{r}_{i}, \mathbf{r}_{j}\right)\right\rangle_{0}=\frac{1}{3}\left\langle\operatorname{Sp} \hat{\alpha}_{2}^{\left(k_{1}, k_{2}\right)}\left(\mathbf{r}_{i}, \mathbf{r}_{j}\right)\right\rangle_{0} \hat{I}, k=1,2
$$

where the angular bracket $\langle\ldots\rangle_{0}$ designates the averaging over the equilibrium distribution function and we suppose that one-ion polarizability is a scalar. The contributions of higher order polarizabilities are relatively small and will be ignored further. Since $\left\langle\mathbf{D}_{0}\right\rangle_{0}=0$, the average $\langle\mathbf{D}\rangle_{0}=0$ can be approximated by the expression

$$
\langle\mathbf{D}\rangle_{0}=n\left[\alpha_{+}+\alpha_{-}+\frac{z}{12}\left(\alpha_{++}+\alpha_{--}+2 \alpha_{+-}\right)\right]
$$

where $z$ is the coordination number and for example $\alpha_{++}=\operatorname{Sp} \hat{\alpha}_{2}^{(+,+)}$is the binary polarizability of two positive ions, which are nearest neighbours $\left(r_{12} \approx a\right)$. Taking into account that the main contribution to $\hat{\alpha}_{2}^{(+,+)}$is caused by the dipole interactions, we can get the characteristic inequality

$$
\alpha_{++} \leq \frac{\alpha_{+}^{2}}{a^{3}} \leq \frac{1}{8} \alpha_{+}
$$

since $\alpha_{+} \sim\left(\frac{a}{2}\right)^{3}$. Therefore, we conclude that the contribution of the binary polarizability in Eq. (A10) cannot exceed $\frac{1}{3}$ of that from the contribution of one-particle ones. 


\section{L. KULINSKII, N. P. MALOMUZH}

To calculate $\left\langle\mathbf{D}^{2}\right\rangle$ at small $\mathbf{E}_{0}$ we use the following assumptions:

1. $\left\langle\mathbf{D}^{2}\right\rangle_{0} \approx\left\langle\mathbf{D}_{0}^{2}\right\rangle_{0}$

2. the dipole moments of ions are strongly correlated only within the region whose size on the average is equal to $r_{s}$;

3. the characteristic dipole moment for this region has order of the dipole moment $\mathbf{d}_{0}$ for isolated molecule $\mathrm{NaCl}$.

As a result we can write

$$
\left\langle\mathbf{D}^{2}\right\rangle_{0} \approx V \frac{d_{0}^{2}}{r_{s}^{3}}
$$

All these estimates allows us to conclude that

$$
\alpha_{\mathrm{eff}} \approx \alpha_{+}+\alpha_{-}+\frac{1}{3} \frac{d_{0}^{2}}{k_{B} T}\left(\frac{a}{r_{s}}\right)^{3}
$$

The values $\alpha_{+}$and $\alpha_{-}$can be approximated by the polarizabilities of $\mathrm{Ne}$ and Ar. Using for $r_{s}$ the estimate obtained above in such a way, we get

$$
\alpha_{-}+\alpha_{+} \ll \frac{d_{0}^{2}}{k_{B} T}\left(\frac{a}{r_{s}}\right)^{3} .
$$

Hence the estimate of the dielectric permittivity and its derivatives with respect to density can be obtained with the help of formulas of Lorentz-Lorenz type:

$$
\frac{\epsilon-1}{\epsilon+2}=\frac{4 \pi}{3} n \frac{d_{0}^{2}}{k_{B} T}\left(\frac{a}{r_{s}}\right)^{3}
$$

The specific form of the relation between dielectric permittivity and the effective polarizability is especially important for the calculation of the derivatives $\frac{\partial^{k} \epsilon}{\partial n^{k}}$. From this point of view the formula of Lorentz-Lorenz type is obtained from first principles and should be considered as physically grounded result. If the dissociation is not complete the formula (A15) takes the form

$$
\frac{\epsilon-1}{\epsilon+2}=\frac{4 \pi}{3} n \frac{d_{0}^{2}}{k_{B} T}\left(\frac{1-\Delta}{2}+\Delta\left(\frac{a}{r_{s}}\right)^{3}\right)
$$

The last term in Eq. (A16) is essential only in the close vicinity of the critical point. In other situations its influence is negligible. Then

$$
\frac{\epsilon-1}{\epsilon+2}=\frac{2 \pi}{3} n \frac{d_{0}^{2}}{k_{B} T}(1-\Delta) .
$$

[1] D. R. Schreiber, M. Conceicano, P. de Lima, K. S. Pitzer, J. Phys. Chem. 91, 4087, (1987).

[2] K. S. Pitzer, Acc. Chem. Res. 23, 333 (1990).

[3] A. Anderko, K. S. Pitzer, Fluid Phase Equilibria 79, 103 (1992).

[4] J. M. H. Levelt Sengers, J. A. Given, Mol. Phys. 80, 899 (1993).

[5] K. S. Pitzer, J. Chem. Phys. 99, 13070 (1995).

[6] M. E. Fisher, J. Stat. Phys. 75, 1 (1994).

[7] G. Stell, J. Stat. Phys. 78, 197 (1995).

[8] H. Weingärtner, M. Kleemeier, S. Wiegand, W. Schröer, J. Stat. Phys. 78, 169 (1995).

[9] Y. Levin, M. E. Fisher, Physica A 225, 164 (1996).

[10] M. E. Fisher, J. Phys.: Cond. Matt. 8, 9103 (1996).

[11] H. Weingärtner, W. Schröer, in: Adv. Chem. Phys., edited by I. Prigogine, S. A. Rice (Wiley, London, 2001), 116, p. 1.

[12] B. Guillot, Y. Guissani, Mol. Phys. 87, 37 (1996).

[13] K. C. Zhang, M. E. Briggs, R. W. Gammon, J. M. H. Levelt Sengers, J. Chem. Phys. 97, 8692 (1992).

[14] F. Hensel, J. Phys.: Cond. Matt. Suppl. A 2, 33 (1990).

[15] M. A. Anisimov, J. Jacob, A. Kumar, V. A. Agayan, J. V. Sengers, Phys. Rev. Lett. 85, 2336 (2000).

[16] J. Jacob, A. Kumar, M. A. Anisimov, A. A. Povodyrev, J. V. Sengers, Phys. Rev. E 58, 2188 (1998).

[17] V. L. Koulinskii, N. P. Malomuzh, V. A. Tolpekin, Phys. Rev. E 60, 6897 (1999).
[18] V. L. Kulinskii, N. P. Malomuzh, Phys. Rev. E 65, 061506 (2002).

[19] R. R. Singh, K. S. Pitzer, J. Chem. Phys. 92, 6775 (1990).

[20] S. Wiegand, R. I. Berg, J. M. H. Levelt Sengers, J. Chem. Phys. 109, 4533 (1998).

[21] S. Wiegand, M. E. Briggs, J. M. H. Levelt Sengers, M. Kleemeier, W. Scröer, J. Chem. Phys. 109, 9038 (1998).

[22] T. Narayanan, K. S. Pitzer, Phys. Rev. Lett. 73, 3002 (1994).

[23] T. Narayanan, K. S. Pitzer, J. Chem. Phys. 102, 8118 (1995).

[24] D. W. Hair, E. K. Hobbie, A. I. Nakatani, C. C. Han, J. Chem. Phys. 96, 9133 (1992).

[25] J. Bischoff, K. Pitzer, Am. J. Sci. 289, 217 (1989).

[26] W. Marshall, J. Chem. Soc. Faraday Trans 86(10), 1807 (1990).

[27] A. G. Moreira, M. M. T. da Gama, M. E. Fisher, J. Chem. Phys. 110, 10058 (1999).

[28] K. S. Pitzer, J. Phys. Chem. 90, 1502 (1986).

[29] K. S. Pitzer, J. L. Bischoff, R. J. Rosenbauer, Chem. Phys. Lett. 134, 60 (1987).

[30] M. Yu. Belyakov, S. B. Kiselev, J. C. Rainwater, J. Chem. Phys. 107, 3085 (1997).

[31] G. Stell, Phys. Rev. A 45, 7628 (1992).

[32] J. M. Caillol, D. Levesque, J. J. Weis, J. Chem. Phys. 
107, $1565(1997)$.

[33] P. J. Camp, G. N. Patey, Phys. Rev. E 60, 1063 (1999).

[34] G. Orkoulas, A. Z. Panagiotopoulas, J. Chem. Phys. 110, 1581 (1999).

[35] P. Debye, E. Hückel, Phys. Z. 24, 195, 305 (1923).

[36] I. R. Yukhnovsky, M. F. Golovko, Statisticheskaya teoriya klassicheskih ravnovesnykh sistem (The statistical theory of classical equilibrium systems) (Naukova Dumka, Kyiv, 1980).

[37] M. E. Fisher, Y. Levin, Phys. Rev. Lett. 71, 3826 (1993).

[38] Y. Levin, X. Lie, M. E. Fisher, Phys. Rev. E 225, 2716 (1996).

[39] L. D. Landau, E. M. Lifshitz, Statisticheskaya Fizika (Statistical Physics) (Nauka, Moscow, 1976), 5, Part 1.

[40] N. Bjerrum, K. Dan. Vidensk. Selsk. Mat. Fys. Medd. 7, 1 (1926).

[41] W. Ebeling, M. Grigo, Ann. Phys. (Leipzig), 37, 21 (1980).

[42] E. Waisman, J. Lebowitz, J. Chem. Phys. 56, 3086 (1972); 56, 3093 (1972).

[43] E. Waisman, J. Lebowitz, Journ. Chem. Phys. 52, 4307 (1970).

[44] Y. Zhou, G. Stell, J. Phys. Chem. 96, 1504 (1992); 96, 1507 (1992).

[45] M. F. Holovko, Condens. Matter. Phys. 2, 205 (1999).

[46] Y. Zhou, S. Yeh, G. Stell, J. Chem. Phys 102, 5785 (1995).

[47] Ph. A. Martin, Rev. Mod. Phys. 60, 1075 (1988).

[48] D. M. Zuckerman, M. E. Fisher, B. P. Lee, Phys. Rev. E 56, 6569 (1997).

[49] P. Minnhagen, Rev. Mod. Phys. 59, 1001 (1987).

[50] N. V. Brilliantov, C. Bagnuls, C. Bervillier, Phys. Lett. A 245, 274 (1998).

[51] J. Hubbard, P. Schofield, Phys. Lett. A 40, 245 (1972).

[52] V. L. Berezinskii, Zh. Eksp. Teor. Fiz. 59, 907 (1970).

[53] J. M. Kosterlitz, D. J. Thouless, J. Phys. C 6, 1181 (1973).

[54] Yu. A. Izyumov, Yu. N. Skryabin, Statisticheskaya mekhanika magnito-uporyadochennykh sistem (Statistical mechanics of magnetically ordered systems) (Nauka, Moscow, 1987).

[55] A. P. Young, Phys. Rev. B 19, 1855 (1979).

[56] J. M. Kosterlitz, J. Phys. C 7, 1046 (1974).

[57] J. B. Kogut, Rev. Mod. Phys., J. Phys. C, 51, 659 (1979).

[58] P. Minnhagen, M. Wallin, Phys. Rev. B 36, 5620, (1987).

[59] B. A. Dubrovin, S. P. Novikov, A. T. Fomenko, Sovremennaya Geometriya. Metody i prilozheniya (Modern Geometry. Methods and Applications, Vol. 1) (Nauka, Moscow, 1989).

[60] T. N. Rice, Solid State Phys. 32, 1 (1977).

[61] Young, Alder, Mol. Phys. 41, 75 (1980).

[62] A. D. Kirshenbaum, J. A. Cahill, P. J. McGonigal, A. V. Grosse, J. Inorg. Nucl. Chem. 24, 1287 (1962).

[63] Spravochnik po dipol'nym momentam veschestv (Reference book on the dipole momenta of the substances), edited by O. A. Osipov, V. I. Minkin, A. D. Garnovskii
(Vysshaya Shkola, Moscow, 1971).

[64] Y. Levin, M. E. Fisher, Physica A 225, 164 (1996).

[65] M. J. Gillan, Mol. Phys. 41, 75 (1980).

[66] V. M. Nabutovskii, N. A. Nemov, Yu. G. Peisakovich, Zh. Eksp. Teor. Fiz. 79, 2196 (1980) [Sov. Phys. JETP 52, 111 (1980)].

[67] V. N. Bondarev, Ukr. Fiz. Zh. 26, 1358 (1981); Phys. Status Solidi 23, 2413 (1981); Zh. Eksp. Teor. Fiz. 82, $2042(1982)$.

[68] V. N. Bondarev, A. B. Kuklov, V. M. Belous, Phys. Status Solidi 31, 42 (1989).

[69] M. E. Fisher, B. P. Lee, Phys. Rev. Lett. 77, 3561 (1996).

[70] W. Schroer, V. C. Weiss, J. Chem. Phys. 109, 8504 (1998).

[71] V. L. Koulinskii, N. P. Malomuzh, Condens. Matter Phys. (Lviv) No. 9, 29 (1997).

[72] T. Poston, I. N. Stewart, Catastrophe Theory and Its Applications, Surveys and Reference Works in Math. 2 Pitman London, 1978.

[73] V. L. Kulinskii, J. Mol. Liq. 105, 281 (2002).

[74] V. I. Arnol'd, A. N. Varchenko, S. M. Gusein-Zade, Osobennosti differenciruemykh otobrazhenii (Singularities of the differential mappings. V. 1) (Nauka, Moscow, 1982).

[75] V. L. Kulinskii, Ukr. Fiz. Zh. (Russian Edition) [Ukr. Phys. J.], 38, 1871 (1993).

[76] V. L. Kulinskii, N. P. Malomuzh, in Proceedings of the Dubna 1996 Conference on $R G^{\prime} 96$ (World Scientific, Singapore, 1997).

[77] H. Frohlich, Theory of Dielectrics: Dielectric Constant and Dielectric Loss, (Clarendon Press, Oxford, 1958).

[78] W.-D. Kraeft, D. Kremp, W. Ebeling, G. Röpke, Quantum Statistics of Charged Particle System (AkademieVerlag, Berlin, 1986).

[79] A. Z. Patashinskii, V. L. Pokrovsky, Fluctuation theory of critical phenomena (Pergamon, Oxford, 1979).

[80] A. Martin, I. Lopez, F. Monroy, A. G. Casielles, F. Ortega, R. Rubio, J. Chem. Phys. 101, 6874 (1994)

[81] M. Neumann, in Water: A comprehensive treatise, ed. by F. Franks (Plenum, New York, 1972).

[82] K. S. Pitzer, J. L. Bischoff, R. J. Rosenbauer, Chem. Phys. Lett. 134, 60 (1987).

[83] K. S. Pitzer, J. C. Tanger, Int. J. Thermophys. 9, 635 (1988).

[84] S. K. Ma, Modern theory of critical phenomena, (W. A. Benlamin, Inc., London, 1976).

[85] A. A. Povodyrev, M. A. Anisimov, J. V. Sengers, J. M. H. Levelt Sengers, Physica A 244, 298 (1997).

[86] V. L. Koulinskii, N. P. Malomuzh, J. Mol. Struct. 381, 199 (1996).

[87] F. J. Wegner, Phys. Rev. B 5, 4529 (1982).

[88] A. Oleinikova, M. Bonetti, Phys. Rev. Lett. 83, 2985, (1999).

[89] R. A. Ferrel, Phys. Rev. Lett. 24, 1235, (1970).

[90] C. M. Sorensen, Chem. Phys. Lett. 117, 606 (1985).

[91] T. Narayan, A. Kumar, Phys. Rep. 249, 135 (1994). 


\title{
ЕФЕКТИ ПОЛЯРИЗАЩЇ ТА КРИТИЧНА ПОВЕДІНКА СИСТЕМ ІЗ КУЛОНІВСЬКИМИ ВЗАЕМОДІЯМИ
}

\author{
В. Кулінський, М. Маломуж \\ Одесъкий національний університет, кафедра теоретичной фізики \\ вул. Дворянсвка, 2, Одеса, Украйна
}

Огляд присвячено критичній поведінці систем із кулонівськими взаємодіями. Особливу увагу приділено вагомості поляризапійних ефектів поблизу критичної точки в таких системах. Виявлено, џо поляризаційні взаємодії суттєво ренормалізують початковий ефективний гамільтоніян систем Ландау-Гінзбурга. Розглянуто двополюсну рідинну модель як базову для творення йонних рідин (розтоплених солей $\mathrm{NaCl}$ ). Показано, що врахування внутрішніх ступенів вільности, зокрема ротаційних, які виникають у йонній парі, дозволяе сформулювати новий підхід до задачі фізичного розміру пари. Подано оцінки критичних параметрів у межах двополюсної рідинної моделі. Запропоновано можливі сценарії критичности системи 3 кулонівською взаємодією. Описано можливість формування неоднорідних станів у критичній ділянщі. Проаналізовано окремі ефекти, поляризащійно спричинені взаємодією між параметром порядку і флюктуацією густини заряду, яка характерна для системи з прямими кулонівськими взаємодіями. Підкреслено деякі нові проблеми, пов'язані з кулонівською критичністю. 